Portland State University

PDXScholar

$5-25-1972$

\title{
An Analysis of change in girls released from Villa Saint Rose
}

\author{
H. Marie Ades \\ Portland State University \\ Kathleen A. Christensen \\ Portland State University \\ Carol L. Parnell Bell \\ Portland State University \\ Shirley A. Groves \\ Portland State University \\ Paul A. Murray \\ Portland State University
}

Follow this and additional works at: https://pdxscholar.library.pdx.edu/open_access_etds

Part of the Educational Sociology Commons, Place and Environment Commons, and the Social Control, Law, Crime, and Deviance Commons

Let us know how access to this document benefits you.

\section{Recommended Citation}

Ades, H. Marie; Christensen, Kathleen A.; Parnell Bell, Carol L.; Groves, Shirley A.; and Murray, Paul A., "An Analysis of change in girls released from Villa Saint Rose" (1972). Dissertations and Theses. Paper 1748. https://doi.org/10.15760/etd.1747

This Thesis is brought to you for free and open access. It has been accepted for inclusion in Dissertations and Theses by an authorized administrator of PDXScholar. Please contact us if we can make this document more accessible: pdxscholar@pdx.edu. 


\section{AN ANALYSIS OF CHANGE IN GIRLS RELEASED \\ FROM VILLA SAINT ROSE}

by

H. MARIE ADES \

CAROL L. PARNELL BELL
KATHLEEN A. CHRISTENSEN

SHIRLEY A. GROVES

PAUL A. MURRAY

A practicum submitted in partial fulfillment of the requirements for the degree of

MASTER OF SOCIAL WORK

Portland State University

1972 
TO THE OFFICE OF GRADUATE STUDIES :

The members of the Committee approve the practicum of H. Marie Ades et a1., presented May 25, 1972 .

Lewis H. Curtis, Chairman

Gack C. Finley

APPROVED :

Dr. Gordoff Hearn, Dean of the Schoo $\overline{l \text { of Social Work }}$

May 26,1972 


\section{ACKNOWLEDGEMENTS}

The research group wishes to express their appreciation to Sister Mary William, and the other Villa saint Rose staff. Their kind cooperation and assistance to us was helpful in preparing this practicum.

We are particularly grateful to Mr. Lewis Curtis, the Practicum Chairman. He has spent considerable time with us and has given us constant support and guidance.

Our thanks to Mr. Jack Finley, member of the practicum committee. His advice and encouragement was beneficial in making this project a possibility. 
TABLE OF CONTENTS

CHAPTER

PAGE

ACKNOWLEDGEMENTS.................. i ii

LIST OF TABLES.................. iv

I INTRODUCTION ................... 1

II $\quad$ METHODOLOGY ...................... 9

Research Design................. , 9

Parents' and Girls' questionnaire... 13

Court questionnaire.............. 16

School questionnaire............. 20

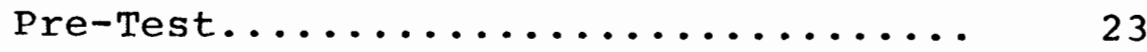

Contact..................... 24

Changes in the questionnaire....... 26

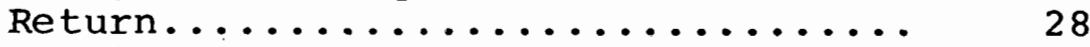

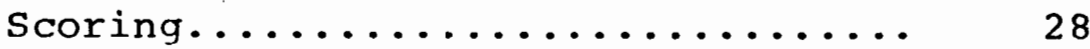

Summary of pre-test............. 32

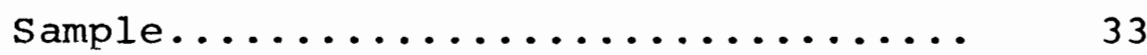

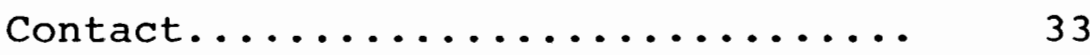

scoring................... 37

III IMPLICATIONS OF DATA............... 40

Summary of Implications of Data....... 55

IV REVIEW OF THE LITERATURE............. 57

Introduction.................. 57

General Theories of Adolescence...... 58

Summary of general theories of

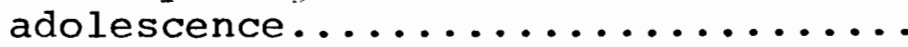

General Theories of Juvenile Delinquency......................

Summary of general theories of delinquency.................

Empirical studies................ 72

Successful treatment program....... 72 
Research on institutions...........

Studies on delinquents released from institutions............... 75

Studies on different causes of

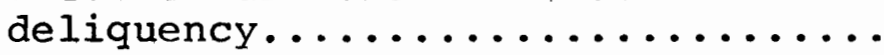

Summary of empirical studies........

CONCLUSIONS .

SUBJECTIVE COMMENTS FROM GIRLS.........

VI

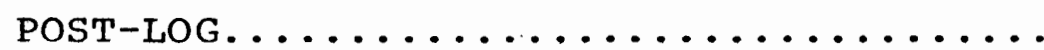

101

Problems with the study............

Recommendations for Villa st. Rose...

Recommendations for Future study......

FOOTNOTES

A SELECTED BIBLIOGRAPHY............

APPENDIX I: GIRL'S CORRESPONDENCE, QUESTIONNAIRE, AND DATA...........

APPENDIX II: PARENT'S CORRESPONDENCE, QUESTIONNAIRE, AND DATA...........

APPENDIX III: COURT'S QUESTIONNAIRE,

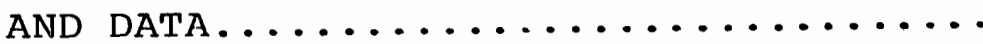


I Comparison of the Total Composite scores on

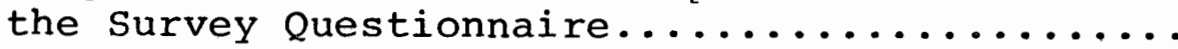

II Comparison of Parent-Daughter Response on Questions 3 and $4:$ Mother............... 45

II Comparison of Parent-Daughter Response on Questions 3 and 4 : Father............... 46

IV Correlations on Specific Family Member Relationships...........................

V Correlations on Responses Regarding the Girl's Experience at Villa Saint Rose. 
CHAPTER I

INTRODUCTION 
When juveniles are defined by society as delinquent they are frequently institutionalized. These institutions are referred to as reform schools, correctional institutions or schools, residential care facilities, treatment centers, or variations of the above. They are state sponsored or privately sponsored. Whatever name is on the sign by the front door, each institution is in the business of "people changing."

All complex organizations use people to pursue their tasks, but people-changing organizations work not only with or through people but also "on" them. People constitute the "raison d'etre" of these organizations, and, as our label suggests, the desired product is a new or altered person. people-changing organizations can be contrasted with organizations that produce, distribute, or service inanimate objects or symbols... In peoplechanging organizations the alterations are the primary end...

People-changing organizations vary in the extent, direction, and difficulty of the change pursued... 1

The excellence of an inanimate product can be measured, weighed, checked, and reproduced; but an altered person is more difficult to measure. If one is in the business of people-changing, it seems important to see if one is in fact changing people. 
This study of post institutional adjustment in one privately sponsored girl's residential care facility is an attempt to look at change in a group of released girls. Villa St. Rose recognizes the need for a continually improving treatment program to better facilitate change. This study is part of a feedback system. The feedback system may aid in program change which in turn may aid in people change.

When the term "treatment" is used to describe a program there is an assumption that the locus of the problem is in the girl. In this study we do not deal with the possibility that the locus of the problem is in American society, its economy, and its institutions.

As we began our study we formulated our tentative purpose as that of evaluating whether girls released from Villa St. Rose demonstrate improved functioning. The girls released from 1968 through 1970 were selected from the tri-county area of Multnomah, Clackamas, and Washington counties. The initial plan was to look at a girl's record at the time of admittance, and then through the use of a questionnaire determine her functioning after release. Her functioning would be judged successful if there was observable improvement (as measured in the scale devised by the study group). We soon found it difficult to operationally define the concepts of success and observable improvement. Because of the inconsistency of information in the files, a "before" measure of the girls was difficult to obtain. Therefore, our questionnaires 
were built around a comparison of behaviors and attitudes before admittance to Villa st. Rose and behaviors and attitudes after release. It was felt success to be a relative and subjective judgment that could be examined in the particular areas of school performance, family relationships, peer relationships, and community adjustment. Questionnaires would be sent to the courts, schools, parents, and girls. With these different perspectives, perhaps some measurement of success could be evolved.

The following is a brief description of Villa st. Rose, its staff, and program. Villa st. Rose exists in response to the need of teen-age girls defined by society as delinquent. Villa st. Rose is administered by a social service oriented order of Catholic nuns, the order of the Good shepherd. Their first "correctional center" was founded in Angers, France in 1829. Villa st. Rose is presently one of 480 Good Shepherd homes throughout the world. It is one of 54 such homes in the United States. It has been operating in Portland, Oregon for about seventy years, serving over 5,000 girls from all parts of the state.

Villa st. Rose can maximally serve 65 girls. Five acres of a possible $111 / 2$ acres are currently in use. The main building is divided into two wings, with convent and social services and administration offices in one wing, and group living areas in the other wing. The group living areas house 15 to 20 girls on each of the three top floors. This is 
dormitory sleeping with an adjacent living room area. On the ground floor the central dining area and kitchen are located. The school facility is connected to this wing and includes a swimming pool. An enclosed yard is behind the building with a tennis and basketball court.

An executive committee composed of nuns is the formal controlling body of Villa st. Rose. Sister Mary William is the present administrator. School staff, group living staff, social services staff, a psychologist, and an activities director all work together to facilitate each girl's fuller functioning. Seven men with Master of Social Work degrees and diversified experience compose the social services staff. Six members of that staff work on a part time basis. One member is the full time resident social worker. Sisters serve as group mothers and are assisted by lay child-care staff.

Girls may be referred to Villa st. Rose by the juvenile court, the Children's Division of the Welfare Department, or private commitment by parents. Girls admitted to villa st. Rose are frequently described as "out of parental control" or "incorrigible". Girls come into contact with the court as run aways, truants, drug abusers, etc.

There was an original emphasis on treatment and rehabilitation, but the means toward this end has been continually changing. In the past religious instruction has been of primary importance. However, at present there is no formalized 
Catholic instruction except as a by-product of living-with and being guided-by sisters. Girls of all and no religious backgrounds are served by Villa st. Rose.

The Villa st. Rose staff has summarized some of its philosophy in a brochure used for staff training:

Problem: Some girls, because of their behavior or family circumstances, are in need of residential treatment.

Philosophy: Each person has infinite value as a human being and deserves the opportunity to achieve her potential.

Goal: Improve each girl's capacity to understand and increase her feeling of self-worth for more personal and social satisfaction.

The high school at Villa st. Rose is fully accredited and cooperates with the programing in surrounding high schools. Through small classes and individual attention the school personnel strive to academically assist each girl toward her potential.

At Villa st. Rose there is the recognition that a girl can change as her environment is changed. The girl's world is now encompassed by the walls and program of Villa st. Rose and selected outside experiences. Her behavior and interactional patterns can be noted. Her ways of expressing warmth, affection, and anger become apparent. A therapeutic community is established with the staff supporting and guiding the girls and the girls supporting each other toward more adequate functioning as individuals in a community. 
It is realized that a girl's post-institutional adjustment is greatly dependent on the parallel adjustment of her family. Mother-daughter groups and family groups are available to expand communication, understanding, and problemsolving skills between mother and daughter and family and daughter.

Peer groups give girls the opportunity to practice new skills in relating interpersonally. The girls discover how they are perceived by others. They experiment with the expression of their feelings in a safe small group.

While participating in individual counseling a girl relates to a concerned adult who encourages her to explore her feelings, talk about her problems, take responsibility for her own actions, set personal goals, and plan for the future.

Within the living unit, a girl can develop new patterns of cooperation. Duties must be shared. This expanded family creates new problems and new opportunities. In group meetings problems are dealt with and plans evolved. A girl becomes part of the living unit that can best add to her growth. The sister responsible for each unit has particular strengths that best serve particular girls. The needs of each individual girl are carefully weighed to determine which available resources might facilitate her fuller functioning

Before each girl is released she meets with members of 
the staff expressing how she perceives herself at that time. She has the opportunity to focus on her plans and goals for the future.

The present transition group home provides selected girls the support and services of Villa st. Rose while they experiment with new freedoms. The group home is located off campus. The girls live in a small group and attend a local high school. A married couple lives in the home giving the loving and limiting of family living. It is hoped that the present group home (and proposed additional group homes) can enhance post-institutional adjustment. As this study began application was being made by the administrator of Villa st. Rose to the Juvenile Delinquency Control and Youth Development officials of the United States Department of Health, Education, and Welfare, to obtain funding for the construction of two additional group homes. One would be used as an additional in-between home for girls soon to be released from the institution. There the girls would have freedom plus the security of group support and continued therapy. The other group home would serve girls judged to be pre-delinquent.

Funding for Villa $\mathrm{St}$. Rose is primarily provided through the Purchase of Care (Program P-5, 1965), ADC-FC (Program 92, 1969), and Joint Funding (1970). Purchase of Care and $A D C-F C$ provide matching state and federal funds for the full operating cost of care which is $\$ 16$ per day 
per girl. Joint funding uses no state funds, but that of the Oregon United appeal matched with federal funds to provide for service costs which are $\$ 14$ per day per girl. 
CHAPTER II

METHODOLOGY 


\section{METHODOLOGY}

\section{RESEARCH DESIGN:}

The original purpose of our research study was to evaluate the treatment program at Villa st. Rose. We felt the best means for accomplishing this would be by evaluating the success or failure of the post-institutional functioning of the girls who had been released from Villa st. Rose. However, after considering the importance of the terms success and failure, we felt it was too judgmental and presumptuous to classify a girl as a success or a failure. Therefore, we decided to modify our research design.

since the focus of our study was on the villa st. Rose program, and the post-institutional adjustment of the girls would be the means of evaluating the program, we felt it adequate to evaluate change within the girl without placing a subjective judgment of success or failure upon her. In order to determine whether there had been a change in her functioning since her release from Villa st. Rose, we found it necessary to place a directional value on this change. This was accomplished by placing change responses on a positive and negative continuum. A positive or negative score would be an indication of the change in the girl's functioning since her release and also indirectly a reflection upon the adequacy of the program at Villa st. Rose. This method of scoring would not reflect upon the sucess or failure of the 
specific girl's functioning.

The girls to be studied would be those released from Villa St. Rose between 1968 and 1970. This three-year time span was chosen arbitrarily. We did want a sufficient amount of time to have elapsed between the release date and the present date in order to allow the girl time to have reintegrated herself into the community.

A past versus present comparison was to be used in determining her functioning. That is, the girl's functioning before admittance to and after release from Villa st. Rose would be measured through the use of questionnaires. These questionnaires would be administered to the girl herself, her parents, her school, and the juvenile court having jurisdiction. The questionnaires were formulated in their entirety by the research team, as it was felt that for our purposes none of the other pre-formulated questionnaires we had considered using were adequate.

When first beginning our research study, we considered using an open-ended questionnaire with which to collect our data. But we decided against this for several reasons. First, an open-ended questionnaire would make it difficult to evaluate the responses, as the liklihood of consistency of the data would be minimal. It would also require that the participants devote a substantial amount of time to completing the questionnaire. We felt this fact would perhaps discourage, and in some cases eliminate, respondents who 
would otherwise have participated in our study. As a result, a fixed-alternative response questionnaire was selected as the instrument of data collection, as it was easier to administer and easier to tabulate the responses. We realized the limitations that this type of questionnaire imposed, such as excluding data which may have appeared in a less structured questionnaire, and limiting ourselves to certain set responses. However, we felt the advantages outweighed the limitations. Thus, we decided to use this type of questionnaire. In designing the girl's questionnaire, we attempted to identify the areas of the girl's environment which might be i.nfluenced by changes in her behavior resulting from her experience at Villa st. Rose. The areas we identified were: the girl's interpersonal relationships with her parents, family, and peers, her school functioning, and her legal involvement with the court.

With the awareness that the girl is functioning in a social environment, we felt it would be interesting and valuable to obtain the perceptions of those she came in contact with to determine whether or not the changes perceived by the girl would be consistent and observable. We felt that the people in the community most capable of observing and evaluating the changes in the girl's functioning would be the parents, school, and juvenile court. Thus, we decided to develop questionnaires for all three in addition to the one 
for the girl.

We considered the girl herself to be the most accurate informational source of her own post-institutional change. She alone was the one to whom the experience occurred, and she could best describe how she believed herself to be presently functioning. Secondly, we would be placing more weight on the parent questionnaire in relation to the court and school questionnaire because of the parents' more intimate involvement with the girl. However, the school usually occupies much of this age group's time and has quite an influence on the adolescent's life. Therefore, we felt that part of the girl's post-institutional adjustment could be reflected in her reported school adjustment. It seemed that the juvenile court could be an important source of information about the girl's legal activities since her release from Villa st. Rose. Also, the court would be an outside observer of the family interaction and social adjustment of the girl, which would provide us with another perspective on the girl's attitudes and behavior.

With these ideas in mind, we formulated the individual questionnaires. Because of the nature of family relationships, we felt that identical questionnaires for parents and girls would be adequate and would serve as the basis for later comparison. A discussion of each of the questions on each questionnaire follows, setting forth our reasons for asking specific questions. 
PARENTS' AND GIRL'S QUESTIONNAIRES :

Question 1. "Are you presently living with your parents?"

"Is your daughter presently living at home?"

Question 2. "Did you live with your parents after your release from Villa st. Rose?"

"Did she live at home after her release from Villa St. Rose?"

These questions were asked for the possibility of later correlation with the attitudinal questions, to determine if the living situation had any effect on the girl's attitudes.

Question 3. "How would you describe your attitude toward your parents prior to your stay at Villa st. Rose?"

"How would you describe your daughter's attitude toward you as parents prior to her stay at Villa st. Rose?"

Question 4. "How would you describe your present attitude toward your parents?"

"How would you describe her present attitude toward you as parents?"

As it was felt that the parents were and possibly still are the main influence on the girl's life, her attitude toward her parents after release as compared to before commitment could be an indicator of how well she is adjusting to life in the community once more. As much of the focus of Villa st. Rose's program is on improving family relationships, the girl would have had a chance to explore further these relationships. This question was designed to determine 
whether Villa st. Rose consistently accomplished this objective.

Question 5. "How would you describe your attitude toward your brothers and sisters prior to villa st. Rose?"

"How would you describe your daughter's attitude toward her brothers and sisters prior to her stay at Villa st. Rose?"

Question 6. "How would you describe your present attitude toward your brothers and sisters?"

"How would you describe her present attitude toward her brothers and sisters?"

A girl's brothers and sisters were felt to play an important role in her life. Therefore, her experience at Villa st. Rose might influence her post-institutional adjustment toward her siblings.

Question 7. "In your estimation how much did you discuss problems with your parents prior to Villa st. Rose?"

"In your estimation how much did your daughter discuss problems with you prior to villa st. Rose?"

Question 8. "In your estimation how much do you discuss problems with them now?"

"In your estimation, how much does she discuss problems with you now?"

The willingness to discuss problems with the parents was taken as another aspect of the girl's emotional involvement with significant others. Another focus at Villa st. Rose is on communication and the importance of being able to communicate 
one's thoughts and feelings. An increased willingness to discuss problems was seen as a sign of good adjustment and as a carry-over of the girl's experience at Villa st. Rose.

Question 9. "To what extent do you feel that your friends had an influence on you prior to villa st. Rose?"

"To what extent do you feel that your daughter's friends had an influence on her prior to Villa st. Rose?"

Question 10. "To what extent do you feel that your friends have an influence on you now?"

"To what extent do you feel that your daughter's friends have an influence on her now?"

Influence of friends was taken to be indicative of peer relationships, which also influence a girl's adjustment. The influence of friends was included as a correlative question to see if there was any comparison between her adjustment since her release from Villa st. Rose and the influence of her peers.

Question 11. "Do you have the same friends now as you did prior to Villa st. Rose?"

"Does she have the same friends now as she did prior to Villa st. Rose?"

The response from this question would be correlated with the responses from questions 9 and 10, as to whether the same or different friends would be responsible for the change or non-change in influence. 
Question 12. "What type of experience do you feel that Villa st. Rose was for you?"

"What type of experience do you feel that Villa st. Rose was for your daughter?"

We felt it would be important to know how the girl felt about her experience at Villa st. Rose. Secondly, her feelings about that experience might be related to her positive or negative post-institutional adjustment.

Question 13. "If you could change something at villa st. Rose, what would you change?"

"About yourself?"

"About the program?"

This was purely informational for Villa St. Rose's benefit.

Question 14. "For those married: How would you describe your present relationship with your husband?"

The girl's experience at villa st. Rose might have an influence on her marital adjustment, as well as on her general post-institutional adjustment.

COURT QUESTIONNAIRE :

Question 1. "What disposition was made by the court following the girl's release from Villa st. Rose?"

Question 2. "What has been the nature of the court's legal involvement with the girl since her release from Villa st. Rose?" 
Question 3. "What has been the pattern of the court's contact with the parents of this girl since her release from Villa st. Rose?"

The first three questions were used to determine what contact and legal involvement, if any, the juvenile court had with the girl and her parents. It appears that one of Villa st. Rose's goals is to prevent future deviant and/or illegal activities by the girls. The amount and extent of contact could be influenced by her prior experience at Villa st. Rose.

Question 4. "What was the parents' attitude toward the court?"

"Prior to commitment to Villa st. Rose?"

"Since her release from Villa st. Rose?"

The parents' attitude toward court involvement could directly or indirectly influence any attempts the court might make in working with both the girl and her family both before and after Villa st. Rose.

Question 5. "What was the parents' attitude toward the girl?" "Prior to commitment to Villa st. Rose?" "Since her release from Villa st. Rose?"

The parents' attitude toward the girl is an important influence on the actions of the girl and could have a large influence on her post-institutional adjustment. The court, as an outside observer, could indicate how they perceived this part of the family relationships. The responses to this 
question would be correlated to the same question on the parents' and girl's questionnaires.

Question 6. "What was the girl's attitude toward her parents?"

"Prior to commitment to Villa st. Rose?"

"Since her release from Villa st. Rose?"

The purpose of this question was to determine how the court, as an outside observer, saw this aspect of the family relationships. Their perception would be compared to that of the parents' and girl's to determine if the change seen by the parents and girls was consistent enough to be observed by the court.

Question 7. "What was the peer group's influence upon this girl's illegal activities?"

"Prior to her commitment to Villa st. Rose?"

"Since her release from Villa st. Rose?"

The peer group influence could either be a positive influence (not favoring or attempting to prevent illegal activity) or a negative influence (approving of, or participating in illegal activities) on her post-institutional adjustment. The purpose of this question was to again compare the court's perception with that of the parents' and girl's.

Question 8. "Upon the girl's release from Villa st. Rose, did she continue in her relationships with the same peer group she had prior to admission to 
Villa st. Rose?"

This adds to the information received from question 7 regarding the peer group's influence upon the girl's illegal activities. If once released from Villa st. Rose the girl continued in her relationship with the same peer group she had prior to admission to Villa st. Rose, and they had a negative influence upon her involvement with illegal activities, it would be indicative of negative post-institutional adjustment. This question was for comparison with question 11 on the parent's and girl's questionnaire, which asks if the girl has the same friends now as she did prior to villa st. Rose.

Question 9. "In your opinion is this girl presently having difficulties as a result of her peer group involvement?"

This question was for our informational purposes. It would not be included in the girl's total composite score.

Question 10. "In your opinion this girl's social-emotional functioning has:"

This question requires the court to subjectively rate the girl's social-emotional functioning since her release from Villa St. Rose. However, the response to this question would not be included in the girl's composite score, but would be for our benefit. 
Question 11. "Do you feel that Villa St. Rose was a very positive experience for this girl?" positive neutral negative very negative

This question asks the court to subjectively rate the girl's experience at Villa st. Rose, as we considered the court to be an additional source of information concerning the girl's functioning. This question would not be included in the girl's total score. However, it would be compared to identical questions on both the parents' and girl's questionnaires.

SCHOOL QUESTIONNAIRE :

Question 1. "What was grade point average for the year prior to her commitment to villa st. Rose?"

Question 2. "What was her grade point average for the year following her release from Vilia st. Rose?"

We felt that a before and after comparison of her school grade point average might be indicative of one aspect of her adjustment to school since her release from villa st. Rose. Information from these two questions would not be included in the girl's total composite score.

Question 3. "How would you rate her attitude toward her teachers and other authority figures for the year prior to her commitment to Villa st. Rose?"

Question 4. "How would you rate her attitude toward her 
teachers and other authority figures in the year following her release?"

It was felt that the relationship between students and school personnel plays an important part in the girl's total adjustment to school. The type of relationship a student has with faculty may have a direct effect upon her studies and upon her attitude towards her school experience. Part of Villa st. Rose's focus is on helping the girl improve her functioning in a school setting. The girl is given the opportunity to experience and re-evaluate her feelings about school. Thus, when she returns to the community, she may take with her some of the attitudes toward authority figures which she experienced at Villa st. Rose. These attitudes may also generalize to other authority figures in her life.

Question 5. "Did she participate in any school club or activities for the year prior to her commitment to Villa st. Rose? If yes, please specify."

Question 6. "Did she participate in any school clubs or activities in the year following her release from Villa st. Rose? If yes, please specify."

Her participation, or non-participation, in clubs or activities, could measure one phase of her total involvement in school. It could also point to the degree of involvement with her peers. Such participation in school activities could be directly related to her functioning in school while at Villa st. Rose. These two questions were used to obtain additional information on the girl's school functioning. 
However, they were not included in her total composite score.

Question 7. "What was her attitude toward her peers in the year prior to her commitment to villa st. Rose?"

Question 8. "What was her attitude toward her peers in the year following her release from villa st. Rose?"

Question 9. "What was the peer group's influence upon this girl's school behavior for the year prior to her commitment to Villa st. Rose?"

Question 10. "What was the peer group's influence upon this girl's school behavior in the year following her release from Villa st. Rose?"

These questions measure the girl's attitude towards her peer group and the peer group's influence upon the girl for the year before and the year following her stay at Villa st. Rose. During adolescent years, the peer group has an important influence on the youth's attitudes and behavior. The girl's attitude toward her peers and the peer group's influence upon her could affect the girl's school adjustment and her relationship with others. Thus, it could influence her postinstitutional adjustment. 
PRE-TEST :

In order to determine the validity, reliability, and usefulness of the questions developed for the study questionnaires, we decided to test it on a small proportion of the population of the girls released from Villa st. Rose from 1968 to 1970. It was necessary to determine if there were any problems with the meaning of words, the forms of the questions, and the questions themselves. It was necessary co structure our questions so that the participants in the study would be able to grasp the meanings we were trying to convey.

Ten percent of the study population was considered sufficient for our purposes. This amounted to six girls, since at this time the projected sample number was set at sixty, which was one-third of the total available population. The pre-test participants were selected at random from among the population of girls released to the tri-county area around Portland during the three years, 1968 to 1970 . These girls were then not included in the sample selected later. Questionnaires were distributed to those-six girls, their parents, the juvenile courts having jurisdiction, and the schools attended by the girls after their release from Villa St. Rose.

Our initial intentions were to interview each girl, her parents, the court counselors, and the school counselors. It was decided that this would be a useful method to employ 
because we felt that individual contact would insure that a greater number of questionnaires would be completed and returned. In addition, this would give us the opportunity of answering any questions concerning the study which the participants might have.

However, it was also felt at this time that the presence of the researcher might introduce a bias into the results, in that the participants might feel pressured to respond in a certain way. So it was agreed upon that the questionnaire would not be administered orally but simply given to the person, who would then fill it out on her own while the researcher waited.

CONTACT :

Preliminary contact was made by sending out an introductory letter (see appendix) to each girl and to her parents, with an explanation of our group and the reasons for and aims of the study. We stated that we would be contacting them by telephone to verify the address and to arrange a time for an interview. One of the parents, when contacted by phone, stated that for personal reasons he did not wish the researcher to come to his house but would be willing to fill out the questionnaire if it were sent out. Another parent at first refused to participate and it was only because of the researcher's detailed explanation over the phone of the contents of the unseen questionnaire that she agreed finally to fill it out, if it were sent out. One parent did agree to 
have the researcher bring the questionnaire out to her house, so this was done. One parent refused to cooperate altogether.

Because of these experiences, for the remaining two parents the questionnaires were sent out after the letters and then a phone call was made to them. This approach worked better and there was much less resistance in cooperation.

The parents were contacted first since these six girls were listed in Villa St. Rose's files as having the same address as their parents. However, the girls were sent out letters and questionnaires in envelopes separate from their parents'. Five girls were contacted this way, even though they all had moved - one to California. For these five their parents supplied the new addresses. One girl had lef: home previously to go to Canada and could not be contacted since her parents had no idea of her specific whereabouts.

Introductory letters were also sent to the juvenile courts in Multnomah, Washington, and Clackamas Counties, explaining our study and asking for their cooperation. The questionnaires were sent along wi.th the letters. Our intertions, as stated in the letter, were to call the court counselors in order to offer further explanations of our study, if necessary, and to arrange a time in which to meet with each counselor and pick up the completed questionnaires. However, within one week the questionnaires had been filled 
out and returned by mail. After discussing this, we decided that the presence of the researcher was not necessary and that for our sample, the questionnaires would be sent out with another letter. In the first letter we had stated that we would be contacting them again at a later date for our sample.

The principals of the schools the girls attended after release were each sent an introductory letter along with a questionnaire. We then contacted them by phone to say that one of our group would be coming out to talk with them. However, problems were encountered from the first. In three cases neither the principal nor the counselor had received the questionnaires. After the situation had been explained, the name of the counselor to whom such inquiries should be sent was obtained and duplicate questionnaires were mailed to them. They did not believe it was necessary for us to come out and stated that they would mail them back.

However, upon the schools' receipt of the questionnaires it was discovered that the interpretation of state senate Bill 160 O.R.S. 44.040 prevented them from disclosing the kind of information we desired. They could only release such information to a parent, or school or agency working with the person at that time. Thus, this development excluded the use of the school questionnaire in our study.

CHANGES IN THE QUESTIONNAIRE :

From the responses to certain questions, it was evident 
that sometimes the meaning we had intended to convey was not coming across. Certain words needed to be changed and alterations made in the forms of the questions. On the parents' and girls' questionnaires, the instructions to "check only one answer" were added in order to avoid the confusion on the part of the respondents as to how many answers should be checked. One answer only would also aid us in tabulating responses. The definitions of those words which we defined were added beside the words themselves, instead of on a separate sheet at the end of the questionnaire. In questions five and six the word "sibling" was changed to read "brothers and sisters" since it was felt that "sibling" is a word little used in everyday vocabulary by most people. Questions five and six were also rearranged in form to better convey the idea that an answer was desired for each sibling and not a composite answer for all of them. On questions seven through ten, the category of "do not know" was added. In order to insure anonymity, the name blanks for the girls on the parents' questionnaire were omitted and the words "your daughter" were inserted.

On all questionnaires, where it was necessary, we rearranged the order of the two parts of the questions - past and present - to insure that the part relating to the past always preceded the part related to the present.

On the court questionnaire, to questions six and seven were added the category of "do not know", since this had been 
left out by mistake. The order of the questions was also rearranged to provide more sequential cohesion.

\section{RETURN :}

A consideration of the return rate on all three types of questionnaires showed it to be quite high. Of the 18 questionnaires sent out, only two were not returned. All 16 returned were complete and able to be scored. On the parents' and girls' questionnaires, 10 out of 12 were returned, for a rate of $83 \%$. It was believed from these results that a good return on the actual sample could be predicted.

SCORING :

Though the pre-test sample was too small to do a statistical analysis of the results, we did score the questionnaires and looked for trends of agreement or similarities among the three questionnaires on each girl. Consideration was also given to inconsistencies in responses.

Using the two scoring techniques explained in the next section of Methodology, we then examined our data. On the parent and girl questionnaires there was a total of 15 points possible. On the court questionnaire there was a total of 13 points possible.

The mean of the parents' composite scores was 4.8 ; however, the range was from a composite of 0 on two questionnaires to $a+12$ on one. The mean of the girls' composite scores was a 5.0 with a range from 1 to 12 . While two parents felt 
there was no change (scores of 0 ) since their daughter's release, all the girls scored themselves on the positive side, indicating some change in a positive direction.

The girls' scores were closely related to those of their respective parents. There was only one point variation in three cases. Though the court composite did not agree closely in score, the direction seemed similar except in one case where the parents scored a zero and the court gave the girl a composite of +12 . The girl herself was in Canada and did not fill out a questionnaire. The composite scores on all three questionnaires were similar in direction of the scoring. The girl and parent questionnaires were very similar. They varied by not more than one point.

There seemed also to be some relation between total points and whether or not the girl returned to her home after her release from Villa st. Rose. The girls who did not return home tended to have lower composite scores than did the girls who did return home for a period of time after their release. This held true for all girls and parents' scores except one girl who is now living in a foster home. She received the highest composite score on both questionnaires. She and her foster parents compared her present living situation with her past home life.

When comparing responses on the individual questions, we found that the girls and parents tended to respond most alike on the girls' attitude toward her parents. They tended to 
agree less on her relationship to her peers and secondly her relationship with her siblings. The parents tended to see more improvement in the girl's relationship to her siblings than the girl did. The parents scored the sibling relationship at least one point higher in every case than did their respective daughter. The court tended to see less improvement in the daughter-parent relationship than did either the parent or the girl.

The final question on all three questionnaires asked the individual completing the questionnaire to indicate his perception of the girl's experience at Villa st. Rose. The alternatives were: Very Positive, Positive, Neutral, Negative, or Very Negative. The parents' and girls' responses co this question were alike in two out of four instances. The court workers agreed with one girl and in no other case was there a similarity in response. Generally there tended to be more similarity among the categories (i.e., parents, courts, girls) than there was in responses concerning each girl. That is, the court responses tended to fall in the middle range around neutral experiences, while the parents' responses tended to be at the extremes. It was either a Very Positive or a Very Negative experience for their daughters. Finally, the girls tended to respond using all five possible responses. When comparing the composite score of each questionnaire to the type of experience each respondent felt the girl had received, there tended to be a relationship between the 
parents' response and the total composite score of their respective questionnaire. That is, those who felt Villa st. Rose had been a very positive experience for their daughter tended to have higher composite scores than did those who felt Villa st. Rose was a very negative experience. This type of correlation did not hold true for either the courts' or girls' responses. 
32

SUMMARY OF PRETEST :

Several structural and grammatical changes resulted from the pretest. These changes were incorporated in the final questionnaires which were used for the actual study.

The findings from the pretest indicated that the questionnaires were both reliable and valid measurements for the purposes of our study. The findings also raised several questions and indicated various trends that need to be given further consideration in evaluating the results of our total study. 


\section{SAMPLE :}

After having administered and evaluated the pre-test, we selected our sample population. The size was set at 30 girls, as it was believed from the pre-test results that this was a sufficient number to provide us with the information we desired. The 30 were selected from a list of 170 girls by the use of a random number table. That is, all the girls were given numbers from 001 to 170 and then corresponding numbers were selected at random from the table. The numbers drawn were not used again. Those girls participating in the pre-test were not considered a part of the sample, since they were from the same list. The girls were from the: tri-county area of Portland: Multnomah, Washington, and Clackamas Counties. This area was chosen since the majority of girls at Villa st. Rose are from these counties. The time limit was set at three years, from 1968 to 1970. This was the same as for the pre-test.

\section{CONTACT :}

Many more problems were encountered in contacting the parents and girls in the sample than had been the case in the pre-test. The addresses and telephone numbers in the files at Villa st. Rose many times were not current. Many people had moved and left no forwarding addresses, making it almost impossible to locate them. Also, resistance was encountered on the part of some people, once located, to filling out the 
questionnaires. Some resistance had been expected, but we had not expected to have so much difficulty in obtaining the present addresses of both girls and parents.

Our original intentions concerning the parents' and girls' questionnaires were to send out introductory letters and then to contact the sample population by telephone to arrange for an interview. However, because of the difficulty encountered in the pre-test with this approach, we decided to change our method. The introductory letters were sent out as planned. If the girls were listed as living at the same addresses as their parents, they were contacted by separate letters. If any of these letters were not returned, we assumed the addresses to be correct and sent out the questionnaires. A telephone call was then made to each person to provide any further explanation that might be necessary concerning the study and to answer any questions the participants had. The call was not made as a first contact to minimize expected resistance, such as was encountered in the pre-test. However, in some cases the telephone numbers were incorrect: and no new numbers could be obtained. Checks for new addresses and telephone numbers were made by contacting the post office, the telephone company, and the personnel at Villa st. Rose. The post office gave us their latest addresses only for us to discover they were not the correct ones. This also occurred at the telephone company. Only in rare instances were we able to locate persons in this manner. 
Generally, such searching usually proved fruitless.

Because of the difficulties encountered in locating our: sample population the predications we had made following the success of administering the pre-test did not hold true. There was an 838 return rate on the pre-test from the parents' and girls' questionnaires. Theoretically, the same percentage should have carried through for the return rate of the questionnaires for the sample population, as the questions and methods of locating the people were essentially the same for the pre-test and for the sample. However, the return rate for the sample was only $37 \%$. The reasons for such a wide disparity are unknown and rather puzzling. The pre-test was considered to be an accurate indicator of how the study could be expected to proceed and of the information which could be obtained. Neither of these expectations was confirmed, for reasons indiscernible to us, as our methods for the two had remained the same. It was very disappointing, as the information thus receired was very minimal.

Problems were also encountered regarding the return rate for the court questionnaires. With the juvenile courts the procedure of contacting the respondants was different from that used with the parents and girls. The questionnaires were sent out first to all three courts with a note explaining that this was the rest of the study group about which we had contacted them earlier for the pre-test. Six questionnaires were sent this time to Washington County and four to clackamas 
County. The counselors at these juvenile courts filled out the questionnaires and returned them to us. The remaining twenty questionnaires concerned girls who had been under the jurisdiction of the Multnomah County Juvenile Court and so were sent there.

However, upon calling the Multnomah County Juvenile Court, a problem was discovered. Some of the court counselors for specific girls were no longer there. As a result, there were no counselors familiar enough with these girls to fill out the questionnaires. However, the Court gave us permission to examine the files of these girls for the purpose of completing the questionnaires. Two researchers and a research advisor went to the court to do this. However, in proceeding this way, we did have a concern that the completion of these particular questionnaires by members of the research group could possibly introduce a research bias into our study. However, since this was the only way information on these girls could be obtained, we went ahead with it. We soon discovered that seven of the wardships had been dismissed upon the girl's release from Villa st. Rose and that no further contact by the court had been undertaken. Thus, it was impossible to complete these questionnaires beyond the first three questions. There were a few court counselors still in contact with the girls, or still at the court who were able to fill out the questionnaires. They then mailed them back to us. 
The total percentage of completed questionnaires from the courts regarding the sample was $38 \%$. This contrasts with the 838 completion rate on the pre-test. Thus, again there is a wide disparity between the predictions made from the results on the pre-test and the actual results on the sample.

\section{SCORING :}

The scoring method was the same for both the pre-test and sample questionnaires. The primary aim was to obtain a numerical composite score on each questionnaire which would indicate a change in the girl's attitudes and behavior after her release from Villa st. Rose when compared to her behavior and attitudes prior to her commitrnent. A composite score of +1 or more was taken as an indication that she had made a change in a positive direction. No change was indicated by a composite score of zero. A change in a negative direction was indicated by a -1 or below.

The composite score was obtained by assigning numerical values to each of the fixed responses on the comparison questions. The responses were arranged with the most positive ones first and the most negative ones last. The responses were then assigned numbers in descending order. mhe most positive response received 5 points while the most negative received only 1 point. The girl was then rated on a particular attitude or behavior before commitment and since her release. She received a positive score if there 
was a more positive response selected on the "since release" part of the question than on the "prior to commitment" part of the question. For example, on the questions: "How would you describe your attitude toward your parents prior to your stay at Villa st. Rose?" and "How would you describe your present attitude toward your parents?", the girl received a plus score if her response on the present-oriented question was more positive than her response on the prior-oriented question. The amount of the score indicated the degree of movement. This scoring method was used on all the comparison questions throughout all three questionnaires.

A different scoring technique was used on the one noncomparison question on the court questionnaire. This second technique was to score one set of fixed alternative responses from $a+5$ to $a-5$, using in-between scores of $+3,-3$, or 0 . The responses were in regard to the nature of the court's legal involvement with the girl since her release from Villa St. Rose. The question was based on fact and was regarded as more serious in nature than were the attitudinal questions. Therefore, we decided that it was more appropriate to assign it a heavier weight, both positively and negatively. Thus, if a girl had had no further contact with the court after release, she received $a+5$ score. If she had been reinstitutionalized, she received a -5 score, as this was regarded as poor adjustment. The other responses received the inbetween scores. 
There were also primarily informational questions which were used only for correlation purposes and were not scored. These included questions $1,2,11,13$, and 14 on the girl's questionnaire; questions 1,2 , and 11 on the parents' questionnaire; questions $1,3,8,9$, and 10 on the court's questionnaire. 
CHAPTER III

IMPLICATIONS OF DATA 


\section{IMPLICATIONS OF DATA}

The return rate on our data was very poor. As a result it is difficult to state with any certainty or assurance that the implications of our findings are generalizable to most girls released from Villa st. Rose. From our sample of 30 girls we were able to contact 17 parents by telephone or mail. Four of those contacted refused to fill out the questionnaires for various reasons. We were unable to contact 13 parents and their daughters either by mail or telephone. They seem to have just disappeared. Neither Villa st. Rose nor the juvenile courts had any knowledge of their whereabouts. Of our total sample of 30 then, we have questionnaires returned from only 13 parents. The girls were even harder to reach since three of the thirteen parents either had no idea where their daughters were or did not know how to reach them by phone or mail. One other girl was in Hillcrest and her parents refused to ask her to fill out a questionnaire. Thus a total of nine girls returned the questionnaire.

The return rate from the three juvenile courts was much better. Questionnaires were returned on all but four girls. Two of those had been private commitments with no court records, the third had been released from Villa st. Rose to a different county and the fourth had run from Villa st. Rose three days after her commitment and has not been seen since. 
Even though the return rate from the courts was better, the information included in them tended to be of little value for our purpose. It appears that the majority of the girls were released from the court's jurisdiction shortly after their release from Villa St. Rose. Thus the courts tended to have no post-release record of the girls. Only eight of the twenty-six court questionnaires returned had any questions completed other than question number two, which pertained to the girl's further legal involvement with the court after her release from villa.

The problems of the poor return rate and the sketchy court data had not been anticipated. On the pre-test sample of six, the return rate on the parent and girl questionnaires had been $83 \%$ or five of each. We were able to contact $100 \%$ or all six parents, though one refused to complete the questionnaire and one did not know where the daughter was living. The courts returned $100 \%$ of the questionnaires and had completed all but one or $83 \%$ of them. The pre-test sample was drawn from the same population as the actual study sample. We have no explanation for the extreme difference between the $83 \%$ return on the pretest and the $37 \%$ on the study. Nor do we know why the courts were able to complete $83 \%$ of the questionnaires on the pretest and only $38 \%$ on the actual study.

Even though the poor return rate makes definite or generalizable conclusions difficult, we were able to make 
some interesting observations. The purpose of the questionnaires was to determine if there had been any changes in the girl's attitudes or behaviors since her release from villa st. Rose. We had decided from the outset that if a girl received a composite score of even plus one $(+1)$, she would be seen as changing in a positive direction as a result of her experience at Villa St. Rose. The composite score is determined by adding all of the final scores of each of the before-admittance after-release comparison questions and obtaining a total. Using the positive composite as the criteria, the results of the parent questionnaires indicate that 678 of the parents or eight out of twelve have seen a positive change in their daughter since her release from villa St. Rose. It is interesting to note that three of the four who have not seen a positive change (they score a composite of zero), are no longer in contact with their daughters, and did not know how to locate them. Eighty-nine percent (89\%) of the parents whose daughters also returned questionnaires felt their daughter had changed in a positive direction. Eighty-nine per cent (89\%) of the girls felt they had changed in a positive direction since their release.

Even though $89 \%$ of both the parents and girls who returned questionnaires had positive composite scores, there was no statistical correlation between the parent-daughter composites. The parents tended to view the change as a much more positive one, that is, they tended to give higher 
composite scores than did their daughters. Table I indicates that the correlation coefficient is 0.54 and that the mean difference is 1.96 points. The absolute mean difference of 3.17 indicates the range when the directionality of the difference is ignored.

TABLE I

Comparison of the Total Composite scores on the Survey Questionnaire

\begin{tabular}{lllr}
\hline Sample & $\begin{array}{l}\text { Parent } \\
\text { Composite }\end{array}$ & $\begin{array}{l}\text { Girl } \\
\text { Composite }\end{array}$ & $D^{*}$ \\
\hline 081 & 7.67 & 3.65 & 4.00 \\
143 & 12.00 & 2.50 & 9.50 \\
153 & 15.00 & 8.00 & 7.00 \\
105 & 6.00 & 9.50 & -2.50 \\
163 & 7.00 & 7.00 & 0.00 \\
102 & 4.00 & 4.00 & 0.00 \\
023 & 2.00 & 3.00 & -1.00 \\
045 & 0.67 & 2.00 & -1.33 \\
133 & 0.00 & 0.00 & 0.00 \\
\hline
\end{tabular}

$\begin{array}{cccl}r^{*}=0.54 & \begin{array}{l}\mathrm{r} \\ \overline{\mathrm{x}}^{\mathrm{d}}=1.96\end{array} \overline{\mathrm{x}}^{\mathrm{d}} & \begin{array}{l}\text { stands for correlation coeffi- } \\ \text { cient } \\ \text { stands for mean difference }\end{array} \\ \overline{\mathrm{x}}^{\mathrm{ab}}=3.17 & \overline{\mathrm{x}}^{\mathrm{ab}} & \begin{array}{l}\text { stands for absolute mean } \\ \text { difference }\end{array} \\ \mathrm{D} & \begin{array}{l}\text { stands for the difference } \\ \text { between parent and daughter } \\ \text { responses }\end{array}\end{array}$

*The four parent questionnaires which were not returned that did not have daughter returns also were omitted as there is no basis for comparison or correlation. 
The parent-daughter pairs tended to agree on the direction of the change. However, the parent tended to perceive the daughter as having changed more than the girl perceived herself as having changed.

One look at the correlation between parent-daughter composites indicates that there is little or no correlation between responses on individual questions in general. There was a strong correlation on one set of questions, however, and that was on Questions 3 and 4 regarding the daughter's relationship with her mother before and after release. Tabie II, (Page 45) indicates that the correlation coefficient on that question was 0.85 with a mean difference of .57 and an absolute mean difference of .86 .

Thus it would seem that there was rather strong agreement between daughter and parent as to the change that had occurred in the mother-daughter relationship. This correlation did not hold for the perceived daughter-father relationship. Table III (Page 46) indicates that the parental response regarding the father on questions 3 and 4 were correlated only at the 0.44 level.

In reviewing the individual parent questionnaires, it seems likely that the majority of the questionnaires were filled out by the mother. Five made specific reference to the father in one way or another in explanation form and were definitely filled out by the mother. The remaining four were not identifiable as one or the other parent. However, since 
TABLE II

\begin{tabular}{lccc}
\hline \multicolumn{4}{c}{ Comparison of Parent-Daughter } \\
& Response on Questions 3 and 4 : & Mother \\
\hline Sample & Parent & Girl & D \\
\hline 081 & 4 & 3 & 1 \\
143 & 4 & 2 & 2 \\
153 & 4 & 4 & 0 \\
105 & 4 & 4 & 0 \\
163 & 2 & 3 & -1 \\
102 & 2 & 1 & 1 \\
023 & 2 & 1 & 1 \\
045 & 0 & 0 & 0 \\
\hline
\end{tabular}

$$
\begin{aligned}
& \mathrm{r}=0.85 \\
& \overline{\mathrm{x}}^{\mathrm{d}}=0.57 \\
& \overline{\mathrm{x}}^{\mathrm{ab}}=0.86
\end{aligned}
$$

Question 3: How would you describe your daughter's/ your attitude toward you as parents/your parents prior to her/ your stay at Villa st. Rose?

Question 4: How would you describe her/your present attitude toward you/your parents? 
TABLE III

\begin{tabular}{|c|c|c|c|c|}
\hline \multirow{2}{*}{ Sample } & \multicolumn{3}{|c|}{$\begin{array}{c}\text { Comparison of Parent-Daughter Response } \\
\text { on Questions } 3 \text { and 4: Father }\end{array}$} & , \\
\hline & Parent & Girl & $\mathrm{D}$ & \\
\hline 081 & -1 & 0 & -1 & \\
\hline 147 & 4 & 0 & 4 & $\therefore$ \\
\hline 153 & 4 & 2 & 2 & \\
\hline 105 & 4 & 4 & 0 & \\
\hline 163 & 2 & 3 & -1 & , \\
\hline 102 & 0 & 2 & -2 & \\
\hline 023 & NA & $\mathrm{NA}$ & - & \\
\hline 045 & 0 & 0 & 0 & 1 \\
\hline 133 & NA & 0 & - & \\
\hline \multicolumn{5}{|l|}{, } \\
\hline & $r$ & $=0.44$ & & ! \\
\hline & $\overline{\mathrm{x}}^{\mathrm{d}}$ & $=0.29$ & & \\
\hline & $\overline{\mathrm{x}}^{\mathrm{ab}}$ & $=1.43$ & & \\
\hline
\end{tabular}

most of the contact that we did have with the parents during the study was with the mother, and since the five that"were identifiable were filled out by the mother, it would appear that probably most, if not all, the others were also filled out by the mother. 
Considering this probability, it seems important to point out that what we appear to have measured is the mother's perception of her daughter's attitudes. The correlation between the mother-daughter relationship is possibly more meaningful because this is the relationship that is shared and experienced by the mother. The father-daughter relationship is one step removed from the mother and she would have to base her response more on observation than on direct experience. Another correlation that lends weight to this trend is in regard to the girl's relationships with her siblings. The correlation coefficient for this response is a negative 0.25 . The hypothesis resulting from this observation is: the more removed the parent is from the relationship he or she is asked to describe, the less likely it is that the response will correlate with the daughter's perception of that same relationship. Table IV indicates the correlations and mean differences with regard to the three relationships.

TABLE IV

Correlations on Specific Family Member Relationships

\begin{tabular}{llllll}
\hline Relationship & $\mathrm{r}$ & $\mathrm{F}_{2}$ & () & $\overline{\mathrm{x}}^{\mathrm{d}}$ & $\overline{\mathrm{x}}^{\mathrm{ab}}$ \\
\hline Mother & 0.85 & 0.80 & 0.02 & 0.57 & 0.86 \\
Father & 0.44 & 0.33 & 0.95 & 0.37 & 1.67 \\
Siblings & 0.25 & 0.11 & 0.79 & 1.21 & 1.67 \\
\hline
\end{tabular}


Thus it appears that though the mother is not as accurate in her perception of the father-daughter relationship as her own with her daughter, the parents' own closer, more intimate relationship provides her with a more accurate perception of the father-daughter relationship than the more distant relationship of sibling to sibling.

It is interesting to note that on all the parent questionnaires, with the exception of one, the "before Villa st. Rose" relationship between mother and daughter was perceived. as antagonistic. This represents 898 of the responses. The "before" relationship between the father and daughter was seen as antagonistic $71 \%$ of the time. The girl perceived the "before commitment" relationship with her mother as antagonistic $55 \%$ of the time and with her father $43 \%$ of the time. There tends to be some agreement between parent and daughter that the relationship with the mother tended to be more often extremely negative than was true with the father. However, there is a rather wide variation between the $89 \%$ and $71 \%$ on the parent questionnaire and the $55 \%$ and 438 on the girls' questionnaire. It appears that the parents tend to perceive the parent-child relationships prior to villa St. Rose commitment as a good deal more negative than did the girl herself. The perception of a more negative attitude on the part of the parents holds true for the sibling question also. The mean response for the girls on the "before commitment" aspect of the sibling question was 3.65 . On the 
response scale used in the questionnaire, that would place her half-way between "tolerant" and"accepting." The mean for the parental response on the "before" of the same question, was 2.2 , or near "indifferent" on the response scale. Thus it appears that the parents tended to perceive their daughters as having more strongly negative attitudes before the commitment to Villa st. Rose than did the girls themselves.

The high percentage regarding the negative or antagonistic attitudes appears to be consistent with the popular held belief that poor parent-child relationships is correlated to delinquent behavior in adolescents. However, in this study, the dissatisfaction in the relationship appeared to be perceived or at least pointed out more often by the parent than by the child.

Questions 7 and 8 dealt with the degree to which the daughter discussed her problems with her parents before and after Villa st. Rose. Again there was no meaningful correlation between the girl's perception and that of her parents. The correlation coefficient was 0.16 , with a relative mean difference of .11 and an absolute mean difference of 1.00 . Neither parent nor the girls tended to respond at either extreme of this scale, however. Individual responses varied from "a great deal" to "not at all" and no one category seemed to predominate with either the before or after responses of the parents. However, $67 \%$ of "before Villa st. 
Rose" responses and $45 \%$ of the "since release" responses on the girls' questionnaires were located at the "very little" or the response scale. The parental range was wider and more evenly distributed.

We also included a question on the amount of positive and/or negative influence peer relationships had on each girl before and after release. Thirty-three per cent of both parent questionnaires and daughter questionnaires stated the girl had the same friends before and after release from Villa St. Rose. However, the agreement between the parent and daughter pair as to who still did or did not have the same friends was 56 \%

The parents indicated that they did not know the amount of influence the peer group had on their daughters on $50 \%$ of: the questionnaires. This was the largest number of no responses on any single question. It appears that the parents do not generally know who their daughter's friends are or how much influence they may exert on her.

Though 89 \% of the girls responded to the peer group question, only $37 \%$ saw the amount of influence exerted by her peers before and after release as changing at all. The influence of peer relationships then, appears to be one area that the parent tends to know little about and one that the girls do not perceive as changing to any significant degree since their release from Villa st. Rose. 
The question on peer group influence was not included in the total composite score. It was included to determine if there was agreement between parent and daughter on the subject and to determine if either felt it was an area of importance. The results tend to indicate that neither the parents nor their daughters consistently perceive it as an area of particular concern.

The rating of the girl's experience at Villa st. Rose was the final question for comparison. The correlation coefficient on this question was 0.45 , with a relative mean difference of 0.55 and an absolute mean difference of 1.00 . Again, there was not enough agreement to be meaningfully related. In percentages the girls rated their experience at Villa st. Rose as follows:

$\begin{array}{lr}\text { Very Positive } & 0 \% \\ \text { Positive } & 338 \\ \text { Neutral } & 22 \% \\ \text { Negative } & 118 \\ \text { Very Negative } & 33 \%\end{array}$

And the parents rated their daughter's experience as follows:

$\begin{array}{ll}\text { Very Positive } & 11 \% \\ \text { Positive } & 338 \\ \text { Neutral } & 338 \\ \text { Negative } & 118 \\ \text { Very Negative } & 118 *\end{array}$

* Percentages do not add to 1008 due to rounding

We also compared the girls' experiences at Villa st. Rose with her composite score to determine if there was any correlation between the higher ratings on the Villa st. Rose 
experience and the higher composite scores. The correlation coefficient was an 0.45. Although this correlation is not statistically significant, it does show a trend toward opposite scores. That is, there appears to be a tendency for girls with low composites to give a higher rating for their Villa st. Rose experience and the reverse is also true. The parental composite was correlated to their Villa st. Rose experience rating at 0.12 and was thus not significant statistically. As is apparent from the percentages above, 668 of the girls tended to perceive their experience on the neutral to very negative side of the scale, while 778 of the parents perceived the experience on the neutral to very positive side of the scale. Thus it appears the parents not only perceived more positive change in their daughters, they also perceived Villa st. Rose to be a generally more positive experience than did their daughters.

The court questionnaires provided us with very little comparative data. The one comparison that we were able to make was in regard to the court's perception of the girl's experience at Villa St. Rose. Table V (Page 53) identifies the numerical responses of the parent, the girl, and the court and compares the correlations on each.

As Table $\mathrm{V}$ demonstrates, the strongest positive correlation, though it was not statistically meaningful, was between the girl and her parents. The responses of the girl and the court were almost significantly correlated in a nega- 
TABLE V

\begin{tabular}{lccc}
\hline \multicolumn{3}{c}{$\begin{array}{c}\text { Correlations on Responses Regarding the Girl's } \\
\text { Experience at Villa Saint Rose }\end{array}$} \\
\hline Sample & Parent & Girl & Court \\
\hline & & & \\
081 & 3 & 1 & NS \\
143 & 4 & 4 & NS \\
153 & 4 & 2 & 4 \\
105 & 4 & 3 & PC \\
163 & 1 & 1 & 3 \\
102 & 3 & 1 & 5 \\
023 & 3 & 3 & 4 \\
045 & 2 & 4 & 3 \\
133 & 5 & 4 & 3 \\
\hline
\end{tabular}

Correlation between Girl and Parents $=0.45$

Correlation between Girl and Respective $=-0.53$ Court

Correlation between Parent and Respective $=0.17$ Court

Numerical Responses:

$$
\begin{aligned}
& 5=\text { Very Positive } \\
& 4=\text { Positive } \\
& 3=\text { Neutral } \\
& 2=\text { Negative } \\
& 1=\text { Very Negative }
\end{aligned}
$$

NS Means No Score

PC Means Private Commitment

tive direction, which means that if the court responded to a specific girl with a positive score, it would be likely that that girl would respond with a neqative score. However, the correlation of -0.53 is not strong enough to make this trend statistically meaningful.

The only other useful data provided by the court questionnaire was the response on question two which asked about 
the nature of the girls' further legal involvement with their respective juvenile courts. As a result of that question, we were able to determine that only seven of the thirty girls in our sample had come into further contact with their respective courts since their release from Villa st. Rose. Two of the seven were re-institutionalized, another received formal court supervision, and the remaining four were referred to the courts again but with no formal action being taken. Thus only 238 of our sample came into further contact with the juvenile court within the tri-county area. However, this figure does not take into account the fact that seven girls were 18 years old at the time of their release from villa st. Rose and were no longer under juvenile court jurisdiction. Nor does the $23 \%$ account for nine of the girls whom we were not able to locate, who were not yet 18 when released from Villa st. Rose. We are assuming that they have moved outside of the tri-county area and thus would be outside of these courts' jurisdictions. After eliminating the girls who are likely to be outside of the juvenile court's jurisdiction for one reason or another, it appears the recidivism rate for this sample could be nearer $50 \%$. 
SUMMARY OF IMPLICATIONS OF DATA:

In summary, as a result of the unanticipated problems of a very poor return rate and inadequate court data, our observations and trends may not be representative of most of the girls released from Villa St. Rose. Indeed the $43 \%$ of our sample that appears to be completely lost within two to four years after release tends to be as representative of our sample as does any of our other observations.

The implications of the questionnaires returned are several. First, $67 \%$ of the parents and 898 of the daughters perceived some positive change in the attitudes and behaviors of the girl since her release from Villa st. Rose. All but one of the parents who did not see a positive change in their daughter have lost contact with their daughter. Thus $89 \%$ of those parents who are still in contact with their daughter perceived positive change.

The parents terided to perceive more positive change in their daughters than did the qirls themselves. However, the parents tended to perceive the girls attitudes as more negative prior to commitment than did their respective daughter:s. Thus the "since release" perceptions may be closer than they appear statistically.

The parental response was meaningfully correlated with their respective dauqhters' response on only one question and that was regarding the girl's relationshin with her mother. The parental response tended to correlate more 
closely with their daughter's responses in regard to the father than to the siblings. The parent tended to respond less frequently to knowledge of their daughter's peer relationships than to any other question. Both parent and girl tended to perceive less change in peer influence than in any other relationship.

Though 898 of the girls felt they had changed in positive direction since their release from Villa st. Rose, only 338 felt their experience at Villa st. Rose was a positive one, while 448 felt it had been a negative or very negative experience. Forty-four percent of the parents felt the experience had been either very positive or positive for their daughters and only $22 \%$ perceived it as a negative or very negative experience.

The court's rating of the girls' experience at Villa $\mathrm{S}$. Rose was not correlated with the girls' own rating nor that of her parents. At a correlation coefficient of -0.53 , the court's rating was almost meaningfully related in the oppositive direction from the girls' rating. The other data provided by the courts was incomplete and provided little information other than that 778 of our sample had not been in further contact with the three juvenile courts included in our study. However, after eliminating those who are outside of these courts' jurisdictions as a result of age or moving, this $77 \%$ is reduced to $50 \%$. That is, $50 \%$ of those who likely could have come into further contact with the dourg did not do so. 
CHAPTER IV

REVIEW OF THE LITERATURE 


\section{INTRODUCTION}

TO THE REVIEW OF THE LITERATURE

Villa st. Rose is involved with adolescent girls who have been referred to them by the juvenile court, the children's division of the welfare department, and private commitments by parents. This led to a review of general theories on adolescence and delinquency, as well as empirical studies which have been done in these two areas.

Problems pertaining to research on institutions, like the difficulties in defining the term "success" were noted. Since the Villa st. Rose study would be a follow-up study, post-institutional research was sought.

Several studies on delinquency show an association between certain variables and post-institutional adjustment. The variables are: home environment, economic status, educational status, religion, and personality factors of the delinquent. An awareness of these variables might influence the Villa st. Rose study.

Section one reviews general theories on adolescence. Section two examines the Oregon Juvenile Code, as well as reviews some theories which have been proposed to explain delinquency. Section three cites institutional follow-up studies, and research which has attempted to explain variables associated with juvenile delinquency 
SECTION I : GENERAL THEORIES OF ADOLESCENCE :

In reviewing the literature on adolescence, we found the scope of the writings to be very broad. With this awareness we chose to select certain well-known theorists within the varying disciplines, and through them provide an overview of the various perspectives on adolescence. By identifying the commonalities among the various perspectives, we hope to acquaint the reader with some of the developmental characteristics of the population being studied.

Defining the term adolescence provides an indication of the process that occurs. The word adolescence is derived from the Latin verb adolescere meaning "to grow up" or to "grow into maturity." In keeping with the definition, adolescence, from a sociological viewpoint, is the transition period from dependent childhood to self-sufficient adulthood. Psychologically, adolescence is viewed as the time in which adjustments must be made from child behavior to adult behavior. In a chronological period, it is viewed as the time from approximately twelve or thirteen to the early twenties, with given individual and cultural differences.

closely related to the concept of adolescence is the concept of puberty. "Puberty" and "pubescence" are derived from the Latin word pubertas, meaning the age of manhood, and pubescere, to reach puberty. Pubescence is the time of physiological development during which the reproductive 
functions develop and mature. Pubescent development on the average takes place in a time span of two years. Although physiological changes occur at all levels of human development, the rate of change is greater during adolescence than any other period in life.

Cultural anthropology, as represented by Margaret Mead, has examined the transition from childhood to adulthood in various societies. In some instances the transition is smooth and without recognition. In other instances puberty rites are used to bring about a transition from childhood to adulthood. Some primitive societies recognize pubescence as the only aspect of the maturation process that they can respect; after puberty young men and women obtain adult status and adult privileges.

In contrast, western society sees a prolonged period of adolescence. In most of the United States, the age twentyone allows certain adult rights and privileges. Aspects of being a minor, such as restrictions on buying liquor, driving a car, marriage, and employment are removed at an earlier age, with variations from state to state. In a legal sense, twenty-one is the termination of adolescence, since at that: time he or she is given legal independence and recognition as an adult.

From a psychological viewpoint, the termination of adolescence is not so much a particular chronological age as it is the degree to which sexual and social adjustment and 
the search for identity are attained.

According to psychoanalytic theory, the stages of psychosexual development are genetically determined and are fairly independent of ervironmental factors. Freud ${ }^{2}$ views in adolescence a close relationship between physiological changes, psychological changes, and one's self-image. During adolescence behavioral changes such as awkwardness and aggressiveness are linked with physiological changes. Physiological changes are also seen as related to emotional changes, especially an increase in emotions such as anxiety, moodiness, and tension.

Erik Erikson ${ }^{3}$ modified the Freudian theory of psychosexual development as a result of findings by cultural anthropoligists. Pubescence, according to Erikson, is characterized by rapid body growth, genital maturity, and sexual awareness. The central concept of his theory of ego development is the acquiring of an ego identity, which is accomplished in different ways from culture to culture. A child, in order to obtain a strong and healthy ego, must receive consistant and meaningful recognition of his achievements and accomplishments.

Erikson ${ }^{4}$ views the peer group, clique, and the gang as assisting the adolescent in finding his own identity in a social context. The strong in-group feelings in adolescent peer groups, with conformity to language and dress, is explained by Erikson as the "necessary defense" against the 
dangers of self-diffusion which exist during this period. 5 Erikson ${ }^{6}$, Mead $^{7}$, and others have stated the major task of adolescence to be the search for a meaningful identity. Mead sees this task more difficult in a modern society than a primitive one. The adolescent is in the process of freeing himself from dependence on his parents, and often views parents as a roadblock to his freedom. As a result, rebellion to parental values and expectations very often occur. Rapid social change, exposure to various value systems, and modern technology often make the world appear to the adolescent too complex and unpredictable to provide him with a stable frame of reference. The push for early adulthood and productivity has made it difficult, in the eyes of many theorists, for youth to establish a stable ego-identity. Friedenberg 8 defines adolescence as the social process through which a clear and stable self-identification is established. As most other theorists, he perceives adolescence as a distinct stage of development, different from childhood and adulthood. This is a time in development when the young person is learning who he is, what he feels, what he can do, and what he can become. In Friedenberg's opinion, this can only be accomplished by setting oneself apart from that culture and breaking the ties of dependency. This process involves conflict. Friedenberg states that adolescence is conflict: 
Adolescent conflict is the instrument by which an individual learns the complex, subtle, and precious difference between himself and his environment. 9 
SUMMARY OF GENERAL THEORIES OF ADOLESCENCE:

The anthropological approach of Mead points to the importance of culture, with its various customs, rituals, or the lack of the same, in determining how and with what degree of ease the child transcends to adulthood through adolescence. Friedenberg, as a sociologist, deals more specifically with the social interactions of the adolescent within a culture. He describes adolescence as a social process and focuses on how the transition to adulthood can be accomplished. The psychological approach, represented here by Freud and Erikson, deals more with the internal states of the adolescent. They describe the physiological and emotional changes that occur within the individual adolescent regardless of the environment which surrounds him.

Though various disciplines tend to view adolescence from different perspectives, there tends also to be some commonalities within them. All the theories reviewed agree that adolescence is a time of physiological and psychological change for the individual. This change is a transitional or maturational process from childhood and dependency to adulthood and independence. Through the process of adolescence the child develops psychologically, physically, and socially to an adult; the major task then, is the establishment of a meaningful identity. The peer group is seen as a major vehicle through which this task is accomplished. 
SECTION II: GENERAL THEORIES OF JUVENILE DELINQUENCY :

Delinquency appears to be a concept about which much is written but little is agreed upon. One of the primary reasons for the lack of agreement among theories of delinquency may be the fact that in our review of the literature we found at least four distinct uses for the term juvenile delinquency. These are:

(a) Delinquency as a deviant behavior.

(b) Delinquency as a distinct legal and/or social status.

(c) Delinquency as a subcultural trait.

(d) Delinquency as a social problem.

Deviant behavior, to state somewhat simplistically, may begin as individual acts, but often develops into maladaptive patterns. These may reflect disorders of personality or rebellion against societal norms and may frequently foreshadow careers in a deviant way of life. Delinquency as a distinct legal and social status may be defined to include predelinquency, adjudicated delinquency, and recidivism. Delinquency as a form of subculture has in the past referred to low-income teen-age subcultures. However, current literature suggests that delinquency may be a phenomenon of the teen-age subculture, irrespective of socio-economic class. Delinquency is seen as a social problem to the extent that it creates widespread public concern. There are continued 
attempts to seek the causes of and solutions to it.

Clifford $\mathrm{Shaw}^{10}$ and associates made a famous and careful analysis of juvenile delinquency in the 1920's and 1930's, showing that the highest rate of juvenile arrests, juvenile court hearings, and correctional school commitments occurred in the lower-class areas of large cities - labeled delinquency areas by Shaw.

Kvaraceus and Millerll present delinquency as a natural outgrowth of lower-class culture. They view it as an attempt on the part of lower-class children and adolescents to conform to patterns of behavior and standards that are acceptec norms of lower-class culture, regardless of the intensity of higher social class disapproval.

Somewhat the same approach has been taken by Ralph England ${ }^{12}$ who has explored the etiology of delinquency in middle-class culture. According to England's theory, the middle-class refuses to recognize the teen-ager in an adult role, and as a result fosters the growth of a teen-age culture that meets many of the needs of adolescents, often in ways not approved by adults.

Cloward and Ohlin 13 have been concerned with lower-class culture and delinquency. They do not see delinquency as an outgrowth of lower-class standards and values, but as a protest of lower-class discontented youth, whose discontent results from an inability to obtain middle-class positions and thus acquire the advantage of middle-class culture. 
In contrast to the sociological writings, many of the psychoanalytic or psychologically orientated writers view delinquency as being closely related to innate individual drives, with cultural and social factors playing secondary roles. The psychoanalytic approach states that every individual is born with a collection of aggressive, primitive, destructive drives. The child is described as being "at war" with the world from the moment of birth. The destructive drives can be tamed by love and understanding in infancy and early childhood. If not, these destructive drives continue to ferment and may form the basis for delinquency and crime. According to this view, the child does not learn the values and behavior patterns of a delinquent sub-culture, but is born a delinquent and must learn how to be a nondelinquent. Often the ego, id, and superego conflicts of the disturbed or delinquent child are a result of inadequate parenting relationships. Because of inadequate parent relationships, treatment is viewed as long-termed, with family involvement necessary. Sidney Berman14, a psychiatrist, states that not until all attempts to treat the family have met with failure, should residential treatment be recommended. Even when residential care is necessary, the focus is directed toward treatment of the child and parents so that when the child is ready to return home, the home is better prepared to meet the child's psychological needs.

Cohen 15 has questioned the accurateness of all juvenile 
delinquent behavior being fitted to a single descriptive theoretical concept or frame of reference. He feels it is better to examine juvenile delinquency with a fresh eye. The delinquency subculture, according to Cohen, holds a negativistic attitude towards the norms of the "respectable" adult society. This is to say the delinquent subculture takes its norms from the larger society, and reverses them. What is judged to be right by the delinquent subculture's standards is considered wrong by the norms of the larger culture. Cohen sees the delinquent subculture as a refuge for those children, for one reason or another, who are denied status in the "respectable" society because they cannot meet: the criteria of the "respectable" status system. The delinquent subculture presents criteria of status which these children can meet.

Charles J. Browning ${ }^{16}$ sees several confusing issues surrounding the nature of delinquency as human behavior. Some of the questions posed by Browning in viewing delinquency as a human behavioral problem are:

Is it, (delinquency), normal or abnormal? Is it necessary to create a pathology to explain it? Is delinquent behavior symptom or syndrome, sickness or disease? Is it a positive thing that can be explained as one of the products of socialization or a negative thing that comes with the failure of social controls to restrain it? Does delinquent behavior constitute a single or more than one dimension? 17

Browning sees these as " only part of a perplexing array of 
points of disputes that confront those who seek to under stand delinquent behavior."18

Most of the girls at Villa st. Rose are referred to the institution by juvenile court. Because of this procedure, it may be useful to review the oregon Juvenile Code with respect to treatment of and outlook toward juvenile offenders. Although the Code was revised in 1969, the girls referred by Juvenile Court to Villa St. Rose from 1967-1968 were done so under the Oregon Juvenile Code of 1959. Thus, the code reviewed below will be the 1959 version.

The primary aim of Oregon's Juvenile Code was and still is to maintain the child in his home with such supportive service rendered to the family as may be indicated:

The provisions of ORS 419.472 to ORS 419.587 shall be liberally construed to the end that a child coming within jurisdiction of the court may receive such care, guidance, and control, preferable in his own home, as will lead to the child's welfare and the best interests of the public, and that when a child is removed from the control of his parents, the court may secure for him care that best meets the needs of the child. ORS 419.474 .19

The Oregon Juvenile Code makes no distinction between delinquency and dependency in the adjudication phase of the proceedings. Neither of the terms "delinquent" or "dependent" are used in the code to describe a child.

ORS 419.476 provides that the juvenile court has exclusive original jurisdiction in any case involving a person who is under 18 and: 
(a) Has committed an act, which, if done by an adult, would be a violation of a law or ordinance;

(b) Is beyond the control of his parents;

(c) Whose behavior, condition or circumstances are such as to endanger his own welfare or the welfare of others;

(d) Is dependent upon a public or private childcaring agency;

(e) Has been abandoned or neglected by parents;

(f) Has run away from home. 20

ORS 419.509 provides a child cannot be placed in a secure custody institution unless the child is found to be within the jurisdiction of the court by virtue of one of the following forms of misconduct:

(a) Violation of a law;

(b) Being beyond the control of his parents, guardian or custodian.

(c) Where his behavior is such as to endanger his own welfare or the welfare of others; (d) Where he is found to be a persistent
runaway. 21 . 
SUMMARY OF GENERAL THEORIES ON JUVENILE DELINQUENCY:

As is evident, there are varying views of delinquency. Shaw, Kvaraceus and Miller, and Cloward and ohlin, speak of delinquency as deviant behavior which they attribute to lower class culture, for one reason or another. Ralph England refutes the theories that define delinquency as lower-class deviant behavior, but rather sees it as a teen-age subculture trait of all classes.

In contrast to the view presented by the sociological approach, the psychoanalytic approach views delinquency as a normal or innate state within the individual youth that comes to the forefront in delinquent behavior because of inadequate socialization.

Cohen and Browning have both attempted to point out the ambiguity of the term delinquency. Their concern seems focused on the hazards of labeling and responding to youths as delinquent when the meaning of such a label is not clear. Delinquent is a vague term, often used as a "catch-all" label, with no identificable meaning behind it. It has been defined as behavior incongruent with society's norms, regardless of whether the motivation for this behavior comes from within the individual or from the environment. It is only when one explores the background of a juvenile that one can accurately determine whether his functioning is maladaptive or not. At that point, behavioral descriptions, along with 
clarification of motivation, are more in order than a general. label.

The girls at Villa st. Rose are not regarded as delinquents, but as individuals with emotional and behavioral problems. Treatment is determined by the needs of the individual rather than by his transgressions against society. 
SECTION III: EMPIRICAL STUDIES:

In the empirical studies section we have attempted to include a wide range of studies which are related to some of the problems and issues which we encountered in the Villa St. Rose study. The problems and issues included can be divided into four areas: 1) a successful treatment program, 2) problems in doing research on institutions, 3) follow-up studies done on delinquents released from institutions, and studies done on different causes of delinquency.

\section{A SUCCESSFUL TREATMENT PROGRAM:}

Dr. Matsushima sheds light on the difficulties in evaluation of successful treatment programs when he states that:

treatment success is defined in various ways. How much change-intrapsychic, in relation to the environment, or both - must there be for a case to be considered successfully treated? What is the relationship between progress in treatment and post-discharge adaptation? If conceptions of treatment success differ from child to child on the basis of specific goals, can one establish a minimal standard common to al1 cases? 22

He goes on to say that:

success in residential treatment is one of the most elusive concepts to define and translate into researchable terms. There is a uniqueness of each child's prognostic base line from which change must be measured. The assumption of a common zero, or beginning point, in all cases is inaccurate. 23 
It is also pointed out that, children who make successful post discharge adaptation may do so because of their families or their post discharge placement situations, rather than because of their progress through treatment. And children who make maximal progress in treatment may do poorly after discharge for similar reasons. 24

Paul Lerman 25 believes that in evaluating institutions it should be realized that some institutions exercise control at intake as to the treatable, and that some delinquents do not complete treatment. This should be noted in counting organizational effectiveness. He says the delinquents not completing treatment are considered statistically nonexistent. It is difficult to think of such outcomes as successes, but organizations do not like to count them as failures. Therefore, they are set aside and ignored. If this group were small, this might be accepted, but it can exceed 30 per cent.

Lerman also maintains that, instead of the success of a program, it might be more relevant to evaluate its failure, and that residential institutions for delinquents are characterized by high rates of potential failure. Researchers interested in evaluating a program should focus on the problem of whether failure rates have been reduced, not whether an institution can claim success. He goes on to propose a humanitarian approach when he says, "it can be argued that even when delinquents are kept for only four months and treated 
closely with their daughter's responses in regard to the father than to the siblings. The parent tended to respond less frequently to knowledge of their daughter's peer relationships than to any other question. Both parent and girl tended to perceive less change in peer influence than in any other relationship.

Though 898 of the girls felt they had changed in positive direction since their release from villa st. Rose, only $33 \%$ felt their experience at Villa St. Rose was a positive one, while 448 felt it had been a negative or very negative experience. Forty-four percent of the parents felt the experience had been either very positive or positive for their daughters and only $22 \%$ perceived it as a negative or very negative experience.

The court's rating of the girls' experience at villa st. Rose was not correlated with the girls' own rating nor that of her parents. At a correlation coefficient of -0.53 , the court's rating was almost meaningfully related in the oppositive direction from the girls' rating. The other data provided by the courts was incomplete and provided little information other than that $77 \%$ of our sample had not been in further contact with the three juvenile courts included in our study. However, after eliminating those who are outside of these courts' jurisdictions as a result of age or moving, this $77 \%$ is reduced to $50 \%$. That is, $50 \%$ of those who likely could have come into further contact with the dourg did not do so. 
CHAPTER IV

REVIEW OF THE LITERATURE 


\section{INTRODUCTION}

\section{TO THE REVIEW OF THE LITERATURE}

Villa st. Rose is involved with adolescent girls who have been referred to them by the juvenile court, the children's division of the welfare department, and private commitments by parents. This led to a review of general theories on adolescence and delinquency, as well as empirical studies which have been done in these two areas.

Problems pertaining to research on institutions, like the difficulties in defining the term "success" were noted. Since the Villa st. Rose study would be a follow-up study, post-institutional research was sought.

Several studies on delinquency show an association between certain variables and post-institutional adjustment. The variables are: home environment, economic status, educational status, religion, and personality factors of the delinquent. An awareness of these variables might influence the Villa st. Rose study.

Section one reviews general theories on adolescence. Section two examines the Oregon Juvenile Code, as well as reviews some theories which have been proposed to explain delinquency. Section three cites institutional follow-up studies, and research which has attempted to explain variables associated with juvenile delinquency 
SECTION I : GENERAL THEORIES OF ADOLESCENCE :

In reviewing the literature on adolescence, we found the scope of the writings to be very broad. With this awareness we chose to select certain well-known theorists within the varying disciplines, and through them provide an overview of the various perspectives on adolescence. By identifying the commonalities among the various perspectives, we hope to acquaint the reader with some of the developmental characteristics of the population being studied.

Defining the term adolescence provides an indication of the process that occurs. The word adolescence is derived from the Latin verb adolescere meaning "to grow up" or to "grow into maturity." In keeping with the definition, adolescence, from a sociological viewpoint, is the transition period from dependent childhood to self-sufficient adulthood. Psychologically, adolescence is viewed as the time in which adjustments must be made from child behavior to adult behavior. In a chronological period, it is viewed as the time from approximately twelve or thirteen to the early twenties, with given individual and cultural differences.

Closely related to the concept of adolescence is the concept of puberty. "Puberty" and. "pubescence" are derived from the Latin word pubertas, meaning the age of manhood, and pubescere, to reach puberty. Pubescence is the time of physiological development during which the reproductive 
functions develop and mature. Pubescent development on the average takes place in a time span of two years. Although physiological changes occur at all levels of human development, the rate of change is greater during adolescence than any other period in life.

Cultural anthropology, as represented by Margaret Mead, has examined the transition from childhood to adulthood in various societies. In some instances the transition is smooth and without recognition. In other instances puberty rites are used to bring about a transition from childhood to adulthood. Some primitive societies recognize pubescence as the only aspect of the maturation process that they can respect; after puberty young men and women obtain adult status and adult privileges.

In contrast, Western society sees a prolonged period of adolescence. In most of the United States, the age twentyone allows certain adult rights and privileges. Aspects of being a minor, such as restrictions on buying liquor, driving a car, marriage, and employment are removed at an earlier age, with variations from state to state. In a legal sense, twenty-one is the termination of adolescence, since at that: time he or she is given legal independence and recognition as an adult.

From a psychological viewpoint, the termination of adolescence is not so much a particular chronological age as it is the degree to which sexual and social adjustment and 
the search for identity are attained.

According to psychoanalytic theory, the stages of psychosexual development are genetically determined and are fairly independent of ervironmental factors. Freud ${ }^{2}$ views in adolescence a close relationship between physiological changes, psychological changes, and one's self-image. During adolescence behavioral changes such as awkwardness and aggressiveness are linked with physiological changes. Physiological changes are also seen as related to emotional changes, especially an increase in emotions such as anxiety, moodiness, and tension.

Erik Erikson ${ }^{3}$ modified the Freudian theory of psychosexual development as a result of findings by cultural anth:opoligists. Pubescence, according to Erikson, is characterized by rapid body growth, genital maturity, and sexual awareness. The central concept of his theory of ego development is the acquiring of an ego identity, which is accomplished in different ways from culture to culture. A child, in order to obtain a strong and healthy ego, must receive consistant and meaningful recognition of his achievements and accomplishments.

Erikson ${ }^{4}$ views the peer group, clique, and the gang as assisting the adolescent in finding his own identity in a social context. The strong in-group feelings in adolescent peer groups, with conformity to language and dress, is explained by Erikson as the "necessary defense" against the 
dangers of self-diffusion which exist during this period. 5 Erikson ${ }^{6}$, Mead $^{7}$, and others have stated the major task of adolescence to be the search for a meaningful identity. Mead sees this task more difficult in a modern society than a primitive one. The adolescent is in the process of freeing himself from dependence on his parents, and often views parents as a roadblock to his freedom. As a result, rebellion to parental values and expectations very often occur. Rapid social change, exposure to various value systems, and modern technology often make the world appear to the adolescent too complex and unpredictable to provide him with a stable frame of reference. The push for early adulthood and productivity has made it difficult, in the eyes of many theorists, for youth to establish a stable ego-identity. Friedenberg 8 defines adolescence as the social process through which a clear and stable self-identification is established. As most other theorists, he perceives adolescence as a distinct stage of development, different from childhood and adulthood. This is a time in development when the young person is learning who he is, what he feels, what he can do, and what he can become. In Friedenberg's opinion, this can only be accomplished by setting oneself apart from that culture and breaking the ties of dependency. This process involves conflict. Friedenberg states that adolescence is conflict: 
Adolescent conflict is the instrument by which an individual learns the complex, subtle, and precious difference between himself and his environment. 9 
SUMMARY OF GENERAL THEORIES OF ADOLESCENCE:

The anthropological approach of Mead points to the importance of culture, with its various customs, rituals, or the lack of the same, in determining how and with what degree of ease the child transcends to adulthood through adolescence. Friedenberg, as a sociologist, deals more specifically with the social interactions of the adolescent within a culture. He describes adolescence as a social process and focuses on how the transition to adulthood can be accomplished. The psychological approach, represented here by Freud and Erikson, deals more with the internal states of the adolescent. They describe the physiological and emotional changes that occur within the individual adolescent regardless of the environment which surrounds him.

Though various disciplines tend to view adolescence from different perspectives, there tends also to be some commonalities within them. All the theories reviewed agree that adolescence is a time of physiological and psychological change for the individual. This change is a transitional or maturational process from childhood and dependency to adulthood and independence. Through the process of adolescence the child develops psychologically, physically, and socially to an adult; the major task then, is the establishment of a meaningful identity. The peer group is seen as a major vehicle through which this task is accomplished. 
SECTION II: GENERAL THEORIES OF JUVENILE DELINQUENCY :

Delinquency appears to be a concept about which much is written but little is agreed upon. One of the primary reasons for the lack of agreement among theories of delinquency may be the fact that in our review of the literature we found at least four distinct uses for the term juvenile delinquency. These are:

(a) Delinquency as a deviant behavior.

(b) Delinquency as a distinct legal and/or social status.

(c) Delinquency as a subcultural trait.

(d) Delinquency as a social problem.

Deviant behavior, to state somewhat simplistically, may begin as individual acts, but often develops into maladaptive patterns. These may reflect disorders of personality or rebellion against societal norms and may frequently foreshadow careers in a deviant way of life. Delinquency as a distinct legal and social status may be defined to include predelinquency, adjudicated delinquency, and recidivism. Delinquency as a form of subculture has in the past referred to low-income teen-age subcultures. However, current literature suggests that delinquency may be a phenomenon of the teen-age subculture, irrespective of socio-economic class. Delinquency is seen as a social problem to the extent that it creates widespread public concern. There are continued 
attempts to seek the causes of and solutions to it.

Clifford Shaw $^{10}$ and associates made a famous and careful analysis of juvenile delinquency in the 1920's and 1930's, showing that the highest rate of juvenile arrests, juvenile court hearings, and correctional school commitments occurred in the lower-class areas of large cities - labeled delinquency areas by shaw.

Kvaraceus and Miller ${ }^{11}$ present delinquency as a natural outgrowth of lower-class culture. They view it as an attempt on the part of lower-class children and adolescents to conform to patterns of behavior and standards that are acceptea norms of lower-class culture, regardless of the intensity of higher social class disapproval.

Somewhat the same approach has been taken by Ralph England ${ }^{12}$ who has explored the etiology of delinquency in middle-class culture. According to England's theory, the middle-class refuses to recognize the teen-ager in an adult role, and as a result fosters the growth of a teen-age culture that meets many of the needs of adolescents, often in ways not approved by adults.

Cloward and ohlin 13 have been concerned with lower-class culture and delinquency. They do not see delinquency as an outgrowth of lower-class standards and values, but as a protest of lower-class discontented youth, whose discontent results from an inability to obtain middle-class positions and thus acquire the advantage of middle-class culture. 
In contrast to the sociological writings, many of the psychoanalytic or psychologically orientated writers view delinquency as being closely related to innate individual drives, with cultural and social factors playing secondary roles. The psychoanalytic approach states that every individual is born with a collection of aggressive, primitive, destructive drives. The child is described as being "at war" with the world from the moment of birth. The destructive drives can be tamed by love and understanding in infancy and early childhood. If not, these destructive drives continue to ferment and may form the basis for delinquency and crime. According to this view, the child does not learn the values and behavior patterns of a delinquent sub-culture, but is born a delinquent and must learn how to be a nondelinquent. Often the ego, id, and superego conflicts of the disturbed or delinquent child are a result of inadequate parenting relationships. Because of inadequate parent relationships, treatment is viewed as long-termed, with family involvement necessary. Sidney Berman ${ }^{14}$, a psychiatrist, states that not until all attempts to treat the family have met with failure, should residential treatment be recommended. Even when residential care is necessary, the focus is directed toward treatment of the child and parents so that when the child is ready to return home, the home is better prepared to meet the child's psychological needs.

Cohen 15 has questioned the accurateness of all juvenile 
delinquent behavior being fitted to a single descriptive theoretical concept or frame of reference. He feels it is better to examine juvenile delinquency with a fresh eye. The delinquency subculture, according to Cohen, holds a negativistic attitude towards the norms of the "respectable" adult society. This is to say the delinquent subculture takes its norms from the larger society, and reverses them. What is judged to be right by the delinquent subculture's standards is considered wrong by the norms of the larger culture. Cohen sees the delinquent subculture as a refuge for those children, for one reason or another, who are denied status in the "respectable" society because they cannot meet: the criteria of the "respectable" status system. The delinquent subculture presents criteria of status which these children can meet.

Charles J. Browning ${ }^{16}$ sees several confusing issues surrounding the nature of delinquency as human behavior. Some of the questions posed by Browning in viewing delinquency as a human behavioral problem are:

Is it, (delinquency), normal or abnormal? Is it necessary to create a pathology to explain it? Is delinquent behavior symptom or syndrome, sickness or disease? Is it a positive thing that can be explained as one of the products of socialization or a negative thing that comes with the failure of social controls to restrain it? Does delinquent behavior constitute a single or more than one dimension? 17

Browning sees these as " only part of a perplexing array of 
points of disputes that confront those who seek to under stand delinquent behavior."18

Most of the girls at Villa st. Rose are referred to the institution by juvenile court. Because of this procedure, it may be useful to review the oregon Juvenile Code with respect to treatment of and outlook toward juvenile offenders. Although the Code was revised in 1969, the girls referred by Juvenile Court to Villa st. Rose from 1967-1968 were done so under the Oregon Juvenile Code of 1959. Thus, the code reviewed below will be the 1959 version.

The primary aim of Oregon's Juvenile Code was and still is to maintain the child in his home with such supportive service rendered to the family as may be indicated:

The provisions of ORS 419.472 to ORS 419.587 shall be liberally construed to the end that a child coming within jurisdiction of the court may receive such care, guidance, and control, preferable in his own home, as will lead to the child's welfare and the best interests of the public, and that when a child is removed from the control of his parents, the court may secure for him care that best meets the needs of the child. ORS 419.474.19

The Oregon Juvenile Code makes no distinction between delinquency and dependency in the adjudication phase of the proceedings. Neither of the terms "delinquent" or "dependent" are used in the code to describe a child.

ORS 419.476 provides that the juvenile court has exclusive original jurisdiction in any case involving a person who is under 18 and: 
(a) Has committed an act, which, if done by an adult, would be a violation of a law or ordinance;

(b) Is beyond the control of his parents;

(c) Whose behavior, condition or circumstances are such as to endanger his own welfare or the welfare of others;

(d) Is dependent upon a public or private childcaring agency;

(e) Has been abandoned or neglected by parents;

(f) Has run away from home. 20

ORS 419.509 provides a child cannot be placed in a secure custody institution unless the child is found to be within the jurisdiction of the court by virtue of one of the following forms of misconduct:

(a) Violation of a law;

(b) Being beyond the control of his parents, guardian or custodian.

(c) Where his behavior is such as to endanger his own welfare or the welfare of others;

(d) Where he is found to be a persistent
runaway. 21 
SUMMARY OF GENERAL THEORIES ON JUVENILE DELINQUENCY :

As is evident, there are varying views of delinquency. Shaw, Kvaraceus and Miller, and Cloward and Ohlin, speak of delinquency as deviant behavior which they attribute to lower class culture, for one reason or another. Ralph England refutes the theories that define delinquency as lower-class deviant behavior, but rather sees it as a teen-age subculture trait of all classes.

In contrast to the view presented by the sociological approach, the psychoanalytic approach views delinquency as a normal or innate state within the individual youth that comes to the forefront in delinquent behavior because of inadequate socialization.

Cohen and Browning have both attempted to point out the ambiguity of the term delinquency. Their concern seems focused on the hazards of labeling and responding to youths as delinquent when the meaning of such a label is not clear. Delinquent is a vague term, often used as a "catch-all" label, with no identificable meaning behind it. It has been defined as behavior incongruent with society's norms, regardless of whether the motivation for this behavior comes from within the individual or from the environment. It is only when one explores the background of a juvenile that one can accurately determine whether his functioning is maladaptive or not. At that point, behavioral descriptions, along with 
clarification of motivation, are more in order than a genera]. label.

The girls at Villa st. Rose are not regarded as delinquents, but as individuals with emotional and behavioral problems. Treatment is determined by the needs of the individual rather than by his transgressions against society. 
SECTION III: EMPIRICAL STUDIES:

In the empirical studies section we have attempted to include a wide range of studies which are related to some of the problems and issues which we encountered in the Villa St. Rose study. The problems and issues included can be divided into four areas: 1) a successful treatment program, 2) problems in doing research on institutions, 3) follow-up studies done on delinquent's released from institutions, and studies done on different causes of delinquency.

\section{A SUCCESSFUL TREATMENT PROGRAM:}

Dr. Matsushima sheds light on the difficulties in evaluation of successful treatment programs when he states that:

treatment success is defined in various ways. How much change-intrapsychic, in relation to the environment, or both - must there be for a case to be considered successfully treated? What is the relationship between progress in treatment and post-discharge adaptation? If conceptions of treatment success differ from child to child on the basis of specific goals, can one establish a minimal standard common to all cases? 22

He goes on to say that:

success in residential treatment is one of the most elusive concepts to define and translate into researchable terms. There is a uniqueness of each child's prognostic base line from which change must be measured. The assumption of a common zero, or beginning point, in all cases is inaccurate. 23 
It is also pointed out that, children who make successful post discharge adaptation may do so because of their families or their post discharge placement situations, rather than because of their progress through treatment. And children who make maximal progress in treatment may do poorly after discharge for similar reasons. 24

Paul Lerman 25 believes that in evaluating institutions it should be realized that some institutions exercise control at intake as to the treatable, and that some delinquents do not complete treatment. This should be noted in counting organizational effectiveness. He says the delinquents not completing treatment are considered statistically nonexistent. It is difficult to think of such outcomes as successes, but organizations do not like to count them as failures. Therefore, they are set aside and ignored. If this group were small, this might be accepted, but it can exceed 30 per cent.

Lerman also maintains that, instead of the success of a program, it might be more relevant to evaluate its failure, and that residential institutions for delinquents are characterized by high rates of potential failure. Researchers interested in evaluating a program should focus on the problem of whether failure rates have been reduced, not whether an institution can claim success. He goes on to propose a humanitarian approach when he says, "it can be argued that even when delinquents are kept for only four months and treated 
with trust, there is no evidence that this "coddling" will yield greater failure rates." 26

PROBLEMS IN DOING RESEARCH ON INSTITUTIONS:

The second area dealt with are the problems in doing research on institutions. Aronson and Sherwood discuss some of these problems when they list these three areas of discord:

1. Pre-occupation of program designers with components of the program without reference to their objectives.

2. Failure of designers to provide an impact model, (changes program was supposed to produce). Researchers had to infer the impact model from the components of the program, or constantly prod the designers to state the theoretical links.

3. Failure of practitioners to cooperate about formation of control groups, provision of records, and adherence to program design. 27

These two authors state that social workers did not understand the necessity for control groups. The social workers state,

Deliberately denying some sufferers of particular condition access to a program designed to alleviate that condition seems like a heartless denial of service.... when are you going to stop experimenting with people, and let us start helping them?28

The practitioner views the information needed in records from a different perspective. His ability to provide service would not be impaired, if all he recorded was the client's 
name. Justification for items such as religion was seen as a violation of client's privacy. This did not help the researchers in their study.

Uneasiness about records also results from the fact that the practitioner often fears that records will be used as a device to measure his ability as a diagnostician, or his judgment in devising treatment methods. There is some validity to this, because it is hard for researchers not to form judgments from such reports.

Aronson and sherwood go on to say that the researchers learned that success in their endeavor requires close cooperation among researchers, program designers, practitioners, and that skill in their craft requires more than technical knowledge. The ability to be diplomatic is important. 29 FOLLOW-UP STUDIES ON DELINQUENTS RELEASED FROM INSTITUTIONS:

The third area pertains to follow-up studies on delinquents released from institutions. One of these studies is a thesis project done on a Good shepherd Home by Sister Mary of st. William. This study was of particular interest for us because Villa st. Rose is also a Good Shepherd Home. Though this study is somewhat outdated--it was written on girls terminated in 1947 and 1948--some of its findings seem worth examining. After a five year interval period, this data was received from a population sample of 189 girls: 
1. More than half of the girls had finished high school before leaving the Home. It is expected that 51.4 per cent found employment within the first six months away from the Home.

2. Almost $1 / 3$ of the number returned to school.

3. Five years later 64.1 per cent of the girls were still in contact with the sisters.

4. 62 per cent of the girls were married, and 42 per cent had children.

5. Of those married, 4.3 per cent were divorced.

6. Twelve girls became Catholic within this five year interval.

7. Fifteen girls resumed delinquency, ten of whom returned to the Home of the Good Shepherd.

8. Three girls were committed to mental hospitals and three others were sent to prison.

9. Two girls died within the five year period. 30

Another thesis done on 500 girls in various homes of the Good Shepherd gives the girls' subjective opinions on what they felt was harmful in their lives, and thus conducive to delinquency. They considered their own character traits before coming to the Good shepherd Home and then compared these past traits with those present at the time of the study. "Over 90 per cent of these girls declared that the knowledge of God and religion was the most powerful force in bringing about the transformation they felt had taken place in their characters." 31

A thesis was done on the subjective experiences of boys entering the community from a correctional school. It sheds light on some of the adjustment problems which confront the delinquent after institutionalization. This study focuses on twenty-three boys released from MacLaren School for Boys in Woodburn, Oregon. The data was collected by interview 
method.

The major findings from this thesis were that the boys had a difficult time adjusting to the community following release, and that they experienced major problems in their dealings with families, schools, and representatives of authority. The boys expressed a desire to become responsible members of the community, but found themselves frustrated to achieve this goal, because society made it so difficult for them. The boys had the largest number of negative experiences after release with their families. They were bitter about being singled out as perpetrators of family arguments. There was evidence that little or no work was done with the boys' families before, during, or after institutionalization. Another of the major areas of difficulty was the boys' re-entry into public schools. Problems in relationships with teachers and fellow students were encountered. Not enough was known about the boys both collectively and individually to enable teachers to handle the variety of problems they presented. The boys felt they were not able to keep up with the demands in the highly structured curriculums of their schools.

Problems with fellow students seemed to stem from feelings of "being on the outside" as the result of institutionalization. Organized efforts to make boys feel a part of the group were lacking. From their reported experiences, the boys were often trying to adjust to what appeared to them an almost 
impossible situation.

Some of the boys were reinstitutionalized within a few weeks after release. The study questions whether this allowed sufficient time for them to make an adjustment. The boys felt that society really had not given them a chance.

Also, many boys designated as having adjusted were still committing acts for which they could have been returned to MacLaren had they been caught. A question asked by the study was, "What is it then in the nature of general behavior which causes one boy to be considered 'adjusted', and another not, even though they are doing the same act?" 32

Another question raised by the DeJardin study is, "What are the attitudes of families, schools, and authorities which places these boys in opposition to certain social institutions? The answer cannot be directed to the boys alone, for they perform satisfactorily in their own peer groups, and their acts in these groups are not all delinquent." 33

A follow-up study was done by James Cowden and Pacht on 152 first admission boys to a correctional school. They cite in their study that "a global prognostic rating based primarily upon family background factors best predicted postrelease adjustment." 34

This study also states that the following personality and behavioral rating factors are predictive of institutional and post-release adjustment. The higher a boy's anxiety rating, the more negative was his institutional and post- 
release adjustment. The higher a boy's score on the MCI scale measuring depressive features, the more positive was his post release adjustment; the higher his guilt rating the more positive the adjustment. The ability to relate positively to adults, as rated by cottage counselors, oroved to be important in predicting both positive institutional and post release adjustment. 35

Another study was done a year earlier by Cowden. He concluded that, "home environment was not found to be significantly related to institutional adjustment or recidivism." 36

It seems important to mention foster homes because many delinquents are placed in a home after institutionalization, and their influence might be important to the child's adjustment.

Taylor and Starr37 did an integrative review of literature on foster parenting, and came up with some interesting conclusions. They found that foster parents recruited are that segment of the population that has minimum education and income. They are family and church oriented. Only 7 out of 45 foster parents were able to use recommended child guidance services in a meaningful way. Of the disciplinary techniques used by foster parents 738 of them were ineffective or harmful. There was also a lack of understanding between the goals of the social workers and the foster parents.

Another study considered the motivations of foster parents, and found that frequently middle-aged foster parents 
want to continue a marital relationship based on parenthood. ${ }^{38}$

\section{DIFFERENT CAUSES OF DELINQUENCY :}

In the fourth area of the empirical studies section different causes of delinquency are examined. The home environment is one of the causes according to Eleanor and Sheldon Glueck. 39 They compare five hundred delinquent boys with five hundred non-delinquent boys, and they found little in these two groups which would point to a definite cause. The most significant factor they determined was that of the home conditions.

Another study considered the effects of family mobility on delinquency. "Past residential mobility as indicated by place of birth of youths $14-17$ years in 1960 , by place of residence of head of household in 1955, and number of years resided in present house did not differ for delinquent and general population. It was not related to the 1960 based delinquency rates of the Los Angeles Youth population." 40 This finding that past residential mobility is not related to delinquency opposes the popular held notion that delinquency is a reflection of recent movement into the larger urban area.

The belief that prolonged separation of a child from its mother during the first five years of life is the foremost cause of delinquent character development was put to test by a research project. A group of 50 school boys who had all 
been separated from their parents and placed in the care of a local Children's Home for over six months at some time in their life, was compared to 50 boys with stable home backgrounds on intelligence, educational, and personality tests. The only significant difference found was higher incidence of enuresis in the Children's Home group. 41

Low economic status had been found to be a main contributor to delinquency. Charles willie's 42 research on delinquency shows that the circumstances of poverty prevents stable home environments. He goes on to state that there is a low rate of delinquency among white youth who are members of a population that has achieved economic affluence. Delinquency rates are similar for whites and non-whites who live in the most disadvantaged environment characterized by many broken homes and low income. Willie believes that community programs should focus first upon increasing the economic status of a population.

Martin Gold43 in his study of Undetected Delinquent Behavior shows a different perspective from Willie's study when he says that:

delinquency begins in the official records of police, courts, and institutions. In his study boys that lived in the poorer parts of town, apprehended by police for delinquency, are four to five times more likely to appear in some official record, than boys from wealthier sections who committed the same kinds of offenses. A statistically inverse relationship was found between class and delinquency, it was extremely slight though with a coefficient of -.12 .44$ 
Simpson and VanArsdol, Jr.,45 looked at the variable of school status and its relative importance in delinquency. It was found that educational achievement, as described by enrollment status, is inversely related to delinquency. A lack of school enrollment is more closely correlated with delinquency.

A comparative study of the delinquent and non-delinquent child, analyzing the variable of religious influence, indicates that church attendence does not bear any significant relationship with delinquency, but that strength of religious feelings, perhaps due to socialization, does significantly differentiate delinquents from non-delinquents.46

Personality factors are another variable that researchers have seen as a cause of delinquency. One study concludes that delinquents have poor self-concepts, and that nondelinquents have good self-concepts. It is suggested that a good self-concept will insulate against delinquency, so work should be done to change the unfavorable direction of socialization and self-concept in those children who are veering toward delinquency. 47 
SUMMARY OF EMPIRICAL STUDIES:

In our review of the literature we found few studies pertaining to post-institutional adjustment. Some of the primary reasons for this lack may be the problems pointed out in the Lerman, Matsushima, and Aronson and Sherwood studies. These studies indicated that in any post-institutional study there are such difficulties as defining "success" in researchable terms, and deciding what is most valid to evaluate about the treatment. One should also try to account for variables such as the family, or post-discharge placement which are not related to the institutional program yet affect the child's post institutional adjustment. Other difficulties include accounting for controlled intake procedures, and institutional dropout which are not included in statistics. Problems exist in accurate record keeping and in dissensions between the practitioner and the researcher. All these problems serve to make accurate, unbiased follow-up studies a problematic, if not an impossible task.

The follow-up studies that we reviewed tended to deal more with the environmental factors which affect a child's post-institutional adjustment, rather than with the effectiveness of the institutional treatment. Such factors as the home environment, the attitudes of the school authorities and peers as well as the community at large are seen as having a significant effect on the adjustment of the child, 
irrespective of his experience within the institutions.

Finally, we reviewed studies which considered factors which may significantly influence a child with regard to delinquent behavior. These studies indicated that such factors as individual home environment, income level, area of residence, school status, religious influence, and individual self-concept can be correlated in some instances with whether or not the child becomes delinquent.

There is, however, disagreement among some of the studies as to conclusions, as exemplified by James Cowden's research. His two studies on the home environment's relation to postinstitutional adjustment of delinquents have contrary conclusions.

Also, in looking over the empirical studies section, it becomes very clear that one cannot really compare one study to another. Different study populations, different variables and biases, and conflict over the meaning of terms regarding operational defintions and the concept of "delinquency", all contribute to the fact that each study is conducted differently and comes up with different results. It is very difficult to know what are the real causes of "delinquency" and what preventative measures can be taken to insure "non-delinquency." The empirical studies section of the review of the literature emphasized the difficult, but also the challenging task of doing follow-up research on an institution. Many of the same problems and issues related here were encountered by the Villa st. Rose study group. 
,

.

'

CHAPTER V

CONCLUSIONS 


\section{CONCLUSIONS}

The purpose of this study was to begin evaluating whether Villa st. Rose as a "people changing" organization is accomplishing its goal. We felt that the best place to begin this evaluation was with the people or as stated in the Introduction "the product" of the "people changing organization". It is our hope that this study will be the first in a series of studies which will ultimately provide Villa st. Rose with a clearer understanding of the strengths and weaknesses in its existing program, and a clearer understanding of the unique qualities and characteristics of the population it serves. I'he final aim will be to provide new program models for incorporating the above into an improved program which in turn will enhance Villa's ability to achieve its goal.

our aim then was to examine and evaluate "the product," or the girls released from Villa, to determine if they had in fact changed in a positive way as a result of their experience at Villa st. Rose.

It is apparent from the files on our sample populatior: and from discussions with Villa st. Rose staff members that the majority of the girls in Villa st. Rose at the time of our sample were referred by the juvenile court as a result of being beyond parental control or engaging in acting-out forms of behavior. There is some indication that this may be changing somewhat since the 1969 code has been written. 
The 1959 Oregon Juvenile Code states that a youth can be institutionalized if he falls within the court's jurisdiction as a result of :

(a) Violation of a law;

(b) Being beyond the control of his parents, guardian or custodian;

(c) Where his behavior is such as to endanger his own welfare or the welfare of others;

(d) Where he is found to be a persistent runaway. 48

Villa st. Rose then, worked primarily with one type of juvenile offender and that is the girl who falls within the second category of the code. There were of course reasons for the selectivity of girls, such as the structure of the facility, the living arrangements for the girls, and the services available. However, for the purpose of this study the importance of this fact is that it provides an identifying characteristic of the population being studied. That is, the girls tended to be acting-out behaviorally or were felt to be beyond parental control.

Another identificable characteristic of our sample is the girls' age range. The girls at Villa st. Rose are adolescents. Adolescence is a period of time when, as pointed out in the Review of the Literature, all girls are going through physiological changes. It is believed that these physiological changes are accompanied by changes in attitude, emotion and behavior as well. The adolescent girl is in a process of maturation. She is attempting a transition from dependence to 
independence and from childhood to adulthood. In order to accomplish this task of "growing up", many changes must necessarily occur. Often, as pointed out by Mead, 49 in this process of achieving independence the adolescent girl perceives her parents as a "roadblock to her freedom". She feels that it is necessary to rebel against their values and expectations, and the stage is set for confusion and conflict. These changes within the girl are often confusing and threatening to the parent as well as the girl. Often neither understand or know how to cope with the changes in feelings, attitudes, and behavior that are occurring. What these changes are and how they are understood and coped with varies with each adolescent and her family. It is important to note however, that the adolescent girl at Villa is like most others her age in that she is trying out new behaviors, experiencing new feelings and developing new attitudes. The resulting tensions, turmoil, and misunderstandings between parent and child are also generally common to all adolescents.

If tension and turmoil are a part of most normal parentadolescent interaction, then why did some girls end up at Villa st. Rose, or any other residential treatment facility? There is no single answer, but our data points to a particular factor which appears to be a possible reason. That is, while tensions exist in most adolescent-parent relationships, it appears that for girls who become institutionalized the parent-adolescent relationship has become one in which the 
parents perceive their daughter responding with predominately extremely negative feelings. In our study, we found that before admittance 898 of the parents perceived their daughter as feeling antagonistic toward the mother, and $71 \%$ perceived her as being antagonistic toward the father. Thus, it appears that a difference between non-institutionalized and institutionalized adolescents could be the extreme degree of angry feelings perceived by the parents of the institutionalized adolescent. Since we had no control group, we can only speculate that, though the normal adolescent-parent relationship is strained and full of tension, the parents do not generally perceive the extreme hostility as did those in our study.

Another factor that our study demonstrates clearly is the importance of the parental perception of the relationship. Only $55 \%$ of the girls felt they were actually antagonistic toward their mother, as compared to 898 of the parents who felt they were. Only $43 \%$ of the girls felt antagonistic toward their fathers, as compared to 718 of the parents who felt they were. Thus, the actual relationship does not seem nearly as important as how the parents perceive it.

It appears then that for most of the girls in our study the new behaviors and attitudes that they began developing during adolescence created or heightened tensions within the parent-child relationship. These tensions ultimately resulted in the parents perceiving their daughter as feeling extremely 
antagonistic toward them. It is likely that perceiving their. daughter as feeling extremely negatively toward them would be quite threatening for parents. The spiraling effect of such perceptions are easy to see. Simply stated, the adolescent begins questioning, resisting, and rebelling against her parents. The parents, feeling confused and threatened, perceive their daughter as angry and react to her as if she were. This in turn results in more aggressive, acting-out behavior on the part of the daughter which further confuses and threatens the parents. Though the interaction is a great deal more complex than described, the basic idea is that the spiraling continues until parent feels that the daughter is totally hostile. By this time she may in fact be hostile. The parent feels that he can no longer understand or control his daughter. Parents are more likely to seek outside assistance when they find that they can no longer control their daughter. Thus, if the girl has not come to the attention of the court through her acting-out behavior, it is likely that the parents will seek the assistance of the court or other agencies. Ultimately some of these girls reach Villa st. Rose.

The implications of our data definitely point to the parent-daughter relationship and even sibling relationships as being more important determinants of delinquency and/or institutionalization than are peer relationships. Erickson, 50 along with many other theorists view the peer group as an integral factor in the adolescent's life. Cohen and others 
describe delinquency in terms of a subcultural phenomenon. Also, it is a popularly held belief that peer group influence is a major contributing factor to the success or failure of a given child, particularly during adolescence. However, our data indicates that only $22 \%$ of the parents and $33 \%$ of the girls felt the peer group had a primarily negative influence on the girl before her admittance to Villa st. Rose. Thus, it would appear that at least $78 \%$ of the parents and 678 of the girls perceived the major problem before admittance as something other than the negative influence of friends. The apparent lack of negative peer group influence on the girls at Villa st. Rose definitely raises serious questions about the accuracy of many existing theories on delinquency, as well as adolescence. However this finding also points to another important consideration.

Some theorists, Cohen ${ }^{51}$ in particular, have questioned the accuracy of trying to fit all delinquent behavior into a single descriptive theoretical concept or frame of reference. Most of the girls in our study came to the attention of the court by nature of their acting-out behavior or by being beyond parental control, rather than as a result of violating laws or other forms of delinquent activity. Thus the most significant implication of this finding appears to be that different types of delinquency cannot be understood or explained within a single frame of reference. There are different influences, causes, and delinquent characteristics 
as well as different types of delinquent behavior. We will gain a more complete and accurate understanding of the "delinquent" and effective treatment models for him when researchers and theorists begin separating "delinquency" by types of behaviors and begin identifying common influences, characteristics, and causes within a specific type.

From the above we can assume then, that extremely negative parent-child relationships and sibling-sibling relationships are significant influences on the acting-out, beyond parental control youth, while peer influence is of questionable significance. This assumption may or may not hold for other types of "delinquent" behavior.

The common characteristics of our study population then were:

(1) They were all females.

(2) They were all within the adolescent age range. As such they were likely involved in the disruptive maturational process normal to this age.

(3) They were defined as delinquent by nature of their acting out behavior, or being beyond parental control.

(4) They tended to be perceived by their parents as feeling extremely negatively toward their parents and toward their siblings to some extent.

(5) Their peers did not appear to be a significant influence on their delinquent status or the institutional placement.

These characteristics have importance in themselves to the degree that they have definite implications for the types of treatment which would be most beneficial for the girls if 
they are to change.

Did Villa st. Rose accomplish its goal? Did the girls in our study change in a positive, or more socially acceptable direction as a result of their experience at Villa st. Rose? At first glance our data looks very encouraging. Though only $69 \%$ of the total parental returns perceived a positive change, $89 \%$ of the parents whose daughters also returned questionnaires felt there had been positive change in their daughters. More importantly, 898 of the girls thenselves felt they had changed in a positive direction. Since we consider the girl to be the most accurate indicator of her own attitudes and behavior, we could say that Villa st. Rose has accomplished their goal with a success rate of 898 . However, in reality, our data demonstrates conclusively only that 898 of the girls have changed. It does not demonstrate conclusively that the change is a direct result of the girl's experience at Villa St. Rose.

The fact that all the girls lived in and received treat-ment through Villa st. Rose was our independent variable. However, there were numerous other independent variables that without a control group, we had no way to account for in our study. Some of these variables include: the normal course of the maturational process itself and the fact that these girls are three years older than when they first entered Villa st. Rose; changes in the girl's living situation; changes in her marital and thus family status; changes in 
parental attitudes, and community attitudes toward the girl. These are all variables which might easily result in changes in the girls after their release from Villa st. Rose. Many of the studies in the Review of Literature stress the importance of the family and home situation, community and school attitudes, as well as the individual girl's personality and emotional make-up in affecting a satisfactory post-institutional adjustment. For example, we do know that of the girls returning the questionnaires only one was still living at home. Does the fact that the girls are generally in a new living situation away from their parents account for their improved attitude toward them or was it a result of their experiences at Villa st. Rose. Some of the girls are married. One may ask if this new relationship has influenced their attitudes and behaviors.

Thus, although our data provides evidence that there has in fact been a positive change in the girls since their release, we were unable to take into account the effects of numerous other independent variables and therefore cannot conclusively demonstrate that the positive change was a result of the girls' experience at Villa st. Rose. The lack of a control group appears to be one of the major weaknesses of our study. However, even with a control group and accounting for the other independent variables we would still have difficulty generalizing our findings because the return on our sample does not appear to be representative of the total 
population.

Four of the parents that we were able to contact refused to fill out the questionnaires or request that their daughters do so. They stated that Villa st. Rose had been a very nega-tive experience for their daughter or that the memory was so painful for all of them that they did not wish to think about it again. These four represent $13 \%$ of our total sample, but $24 \%$ of those we were able to contact. Secondly, $43 \%$ of our sample had apparently completely disappeared. We were unable to reach them by mail, or telephone and neither Villa st. Rcse nor the court had any knowledge of their whereabouts. The longest possible period since the release of an individual girl is three years, yet $43 \%$ of parents and an additional $13 \%$ of the girls were lost for all practical purposes. The implications of this finding will be discussed shortly, but for the moment its importance lies in the fact that the total return rate for both the parents and the girls was $37 \%$. When considered separately it was $30 \%$ on the girls' questionnaires and $43 \%$ on the parents. The percentage that was lost then, is almost as great as those who returned questionnaires or refused to respond. Thus considering the representativeness of our returns, it would seem to be as accurate to state that girls and their families tend to disappear as a result of the Vilia St. Rose experience as it is to state that girls change in a positive direction as a result of that same experience. The high mobility rate of our samole is perplexing. 
Simpson and VanArsdol, Jr. 52 found, in 1955, that residential mobility was not related to delinquency. That is, the number of years families lived in one home did not differ for familjes of delinquents when compared with those of the general population. Also, on our pre-test we were able to locate $100 \%$ o: the sample. However, our findings on the study seem to disagree with the Simpson and VanArsdol, Jr., findings. Fortythree percent of our sample had moved at least once in the past three years. The information provided us by the postal service indicated that many had moved two or three times in three years.

Neither Villa st. Rose nor the girls' respective juvenile courts have made much of an attempt to follow up on the girls since their release. It seems likely that some form of follow-up work with the girls and their families would have prevented such a drastic loss of contact with families for research purposes. This however is a separate issue from the possible therapeutic effect that follow-up services might provide. In due respect to Villa st. Rose, it seems appropriate to mention that we are aware that there are numerous difficulties involved in follow wo, such as lack of staff and the loss of jurisdiction over the girl once she is released and referred back to the court. However, for many reasons, including the continual evaluation of their program and the therapeutic effect on the girl and her family, Villa st. Rose needs to make every attempt to develop a follow-up 
program.

After considering the major weaknesses of our study, that is, the lack of a control group which could have enabled us to account for the numerous variables which were not accounted for and the non-representative return from our sample, we are able to conclude only that there is a high degree of agreement that girls released from Villa st. Rose change their attitudes and behaviors in a more socially acceptable direction. Though the parents tend to perceive the change as being more positive than do their daughters, there is definite agreement on the direction of the change.

The limited information that we were able to obtain from the juvenile courts indicates positive change also. That is, before their experience at Villa $s t$. Rose all but two girls, or $93 \%$ of our sample, had come into contact with the court. However, since their release only $23 \%$ or seven girls have come into further contact with the court. Only two of those were re-institutionalized. It appears that the offenses of at least four were rather minor since no formal action was taken. As demonstrated in Chapter III, these seven may actually represent 50 \% of the girls who could have come under the tricounty juvenile courts' jurisdiction. However, we have no way of determining the exact number of girls who were outside of the court's jurisdiction.

Our data is insufficient to conclude that the positive change is a direct result of the girl's experience at Villa 
st. Rose. Our final factor which may be an indication that many of the girls and their parents do not perceive the change as a result of Villa st. Rose's program is their evaluation of that experience. Sixty-six percent of the girls felt their experience at Villa had been "neutral" or "very negative". of that $66 \%, 33 \%$ felt that it was a "very negative" experience. Not one girl perceived Villa st. Rose as a "very positive" experience, though 338 did see it as a "positive" experience. There was a wider range of parental responses. However, 55\% of the parents indicated that they felt Villa had been a "neutral to very negative" experience for their daughters. It is interesting to compare the juvenile courts' responses to this question. While they responded to this question on only half of the girls' questionnaires, the responses they did make were $50 \%$ "positive to very positive" and $50 \%$ "neutral". They did not perceive Villa st. Rose as being a "negative" or "very negative" experience in even one instance. As mentioned in Chapter III, the courts' responses were almost significantly correlated in a negative direction to the girls' responses. That is, if the court felt the experience to be positive, you could almost say with certainty that the girl would perceive the same experience as negative. Since over half of the courts' responses to this question were on questionnaires they were unable to complete because of a lack of information on the particular girl, it seems likely that the courts' responses are more indicative of agency relations or the courts' 
expectation of the Villa st. Rose experience rather than the actual experience of a particular girl at Villa st. Rose.

The question on the girl's experience at Villa st. Rose was designed to determine the extent to which the girl, her parents, and the court, perceived changes in the girl as resulting from her stay at Villa st. Rose. (See Appendix, question 12). The implication is then, that 668 of the girls and 55\% of their parents perceived the experience as resulting in no change or as decreasing the girls" ability to "cope with herself and the community." Thus the positive changes observed by these respondents is not perceived as being related to the Villa st. Rose program.

The meaning of these figures for Villa st. Rose is not clear. Experiencing confinement, living away from home, living with other girls, (many of whom have behavioral and emotional problems and all of whom have social adjustment problems), is at best difficult, particularly during adolescence. However, when a girl or her parents look back on the experience from one to three years later and still do not perceive it as an experience that was helpful, one begins to ask why. Is the concept of institutional treatment invalid? When we as a community institutionalize youth, are we helfing them or protecting ourselves? Or, is it that the concept of institutional treatment is valid and villa st. Rose just needs to evaluate and improve on its existing programs? or is it that even though institutional treatment is not $100 \%$ 
pleasant or successful, it does often prevent further adoles-cent and/or family deterioration and thus prevents more serious problems? There were $33 \%$ of the girls and 448 of the parents who felt Villa st. Rose was a positive, helpful experience. Thus Villa st. Rose and its institutional treatment have been helpful for some, and therefore we must not perceive institutional treatment or Villa st. Rose as "all" bad. However, as long as over half of those who are involved on the receiving end do not perceive the experience as helpful or of value, the necessity for continued questioning and critically evaluating the existing treatment programs persists.

\section{SUBJECTIVE COMMENTS FROM GIRLS:}

When designing the girls' questionnaire, we felt it of importance to allow the girl space in which to express any changes she might have wanted implemented at Villa st. Rose while she was there, either within herself, or with the program at the institution. It is with the aim of further evaluation of Villa st. Rose's program that we have included the following comments written by some of the girls in our sample:

"I suggest investigating the real reason for confinement. Make sure they [the girls] really need correction."

"I would have stood up for myself against anyone. Instead of letting them tell me who I was - I should have told them."

"We got to see our parents only twice a month and when I got out I felt as if I hardly knew them." 
We could not read newspapers or correspond with friends. When I got out I felt out of touch - it was like a different world in there."

"The program then, was more rigid than it is now. The thing I think was most important was privacy. There just wasn't too much time to think alone. Also there was a feeling of hystericalness - anytime anything happened to a girl personally, or a friend, whether it was something within the school or outside, she cracked up. It didn't happen all the time - but things were too high strung. There should be a controlling calmness instilled."

"Unlock the doors, take the bars off the windows, more trust, more outings..."

"More freedom to express opinions..."

"As far as school goes, it was not teaching relevant subjects, and the students had no choice on what courses they wanted to take."

"The diet was too starchy, and we had little exercise." 


\section{CHAPTER VI}

\section{POST-LOG}


POST-LOG

PROBLEMS WITH THE STUDY:

One of the problems in the study was that of trying to define the concepts of "sucess" and "failure" for measurement. As has been noted in the Methodology chapter, we decided that these could not be adequately defined for the purposes of our study. "Success" or "failure" for the girl as Dr. Matsushima 53 points out, would have to be individually calculated from changes from her own baseline and could not be a global concept applicable to everyone. To have specific goals applicable to everyone would result in a loss of flexibility and response to individual needs by the program in order to fit everyone to a desirable mold. But to have a program individually tailored to each girl means that only very general goals can be formulated for the entire program, while specific goals must be designed at the entrance of each girl to best meet her needs. Therefore, to define the "success" or "failure" of each girl, one would have to take into account her needs, capacities, and behavior as they were seen at the time of her entry and again later at a designated time to compare the change.

Thus, we fully realize that it is extremely difficult to have specific goals applicable to the whole population of girls. At the same time this situation makes it very hard for researchers to measure success of the program, except by studying specific cases in detail. As Aronson and sherwood 54 
point out, the researchers usually have to infer goals of the program from its different parts, since no impact model is provided. While there may be a realistic basis for having no model, we did have to infer from the general goals and from components of the program what some of the more specific goals were, such as improving family relationships, improving peer relationships, and providing a positive school experience, in order to know what types of questions to include in our questionnaires. This was difficult to do. If such an impact model could be devised by the practitioners, it would be much easier for researchers to measure the effectiveness of the program and would also provide more accurate and more useful results.

A control group was not used in our study because of the time and difficulties involved in selecting such a group. For instance, the age grouping would have had to be the same for both the control and the treatment group. But the control group would have had to be a group of girls who had been involved with legal authorities and had recorded emotional or behavioral problems but had not been put into a residential treatment setting. The enlisting of the cooperation of those involved in obtaining this group without having any authority behind us for this could have been a problem. Although we realize that these limitations undoubtedly could have been overcome, our group did not feel that there was a sufficient amount of time for our study to have such a broad scope. 
That the lack of a control group does place a limitation on our study is clearly understood. Because there is always the possibility that girls not at Villa st. Rose could have evidenced the same kinds of attitudes and the same kinds of changes in those attitudes as those girls who had been at Villa St. Rose, we cannot state for certain that the changes shown were due to the program at Villa st. Rose. Independent variables, such as maturational process, economic and educational status, religious beliefs, living situation, and personality factors, also were not taken into account and thus may have influenced the changes that were perceived in the girl.

Another limitation to the study is that of the subjective judgments involved in filling out the questionnaires. The only truly objective parts are those questions on the court questionnaire which ask whether the girl has had further legal involvement with the court. The rest involved questions of comparison concerning the girl's present functioning versus her functioning previous to villa st. Rose. However, these questions measured more accurately how the girl herself and others perceived her to be functioning. This could be different from how she is functioning in reality. But the problem arises here of what exactly is reality, and how that can be measured? It appears that what is taken for reality is really composed of everyone's perception of what that "reality" is. It is difficult to 
make a completely objective decision, without its being colored by biases and prejudices. Taking this into account, what we measured then may indeed be the reality of the situation.

The limitation imposed by using comparison questionnaires was that if one questionnaire were missing, such as a parent questionnaire, there was a restriction on the amount of information gathered, and thus on our ability to make an adequate assessment of the actual improvement of the girl, since we had nothing with which to compare responses. It would have been better, it seems at this point, to have designed questions which would have individually given us all the information necessary for our purposes, without the necessity of comparison. Thus, had one questionnaire not been received, it would not have had such a detrimental effect on the actual results. More data would have been available to measure the chanqe in the girl since her release.

The method of sending out questionnaires versus interviewing has already been partially discussed in the Pre-Test section of the Methodology chapter. Essentially, we had several objections to interviews. We felt that interviews were more threatening to the participants. Some people did not want to be reminded of memories connected with villa st. Rose and felt it would be disruptive to the family structure for an interviewer to come to the home. However, these 
individuals had less objection to our sending of the questionnaire by mail. Also, interviews were seen by some participants as being less anonymous. Distance was another factor. Some people were unavailable to interviewers simply because they were too far away. For instance, two were in California, and another was in Canada. Lastly, it would have been more costly in terms of time and money for the researchers to do interviews.

Problems were encountered, as has been noted, in locating people for the sample. The same did not occur with the pretest, but the reason remains unknown. Part of the problem was with the paucity of material needed for research purposes in the files at Villa st. Rose, such as current addresses and telephone numbers. However, this difficulty did not occur with the pre-test population. The sample appeared to be a more mobile population and to have less contact with Villa st. Rose than the pre-test population appeared to have. We can only speculate that part of the reason for less contact was that, for whatever reason, the parents and girls did not desire to have any further contact with villa st. Rose. Since little or no follow-up by villa st. Rose was done, it is probable that the parents and girls did not take the initiative to make contact either. There is the possibility that informal contact was made but not recorded in the file. This left the researchers without up-to-date information on many girls. 
After having experienced the difficulty of making contact with much of the population in a follow-up study, the researchers wonder if it would not have been better to have chosen a captive population for study. For instance, a questionnaire could have been developed for use with the girls presently residing at Villa st. Rose. This would undoubtedly have given us much more data to work with and perhaps provided more useful information.

One minor point concerning the questionnaires has to do with the timing of their mailing. The sample population's questionnaires were mailed the month previous to Christmas. The idea that the questionnaires could have been mixed up and forgotten among the Christmas mail has occurred to us. When contacted a second time, a few parents did reply that they had the questionnaires around the house and were going to return them, or that they had lost them and so requested another. Whether this actually occurred because of Christmas can not be confirmed.

RECOMMENDATIONS FOR VILLA ST. ROSE:

One of the recommendations we would like to make for Villa st. Rose is that the method of keeping files be more orderly and that the information be as current as possible. This current information, or any change in information, should be located at the very front of the file on a separate sheet. This would eliminate the need to read letters in the file in order to search for current addresses or telephone numbers. 
For Villa st. Rose's purposes the files may be adequate, but for research purposes they are not. Even the specific treatment programs for girls, had we chosen to do that study, were not clearly defined as to the specific goals.

Another recommendation would be that Villa st. Rose attempt to do some kind of follow-up with the girls after release. We realize that because of shortage of staff and money this might be difficult. However, not all girls would need further contact. A few could be periodically selected for such a purpose. If they were told they would be followedup, this might make contact and cooperation easier. Such follow-up would help Villa st. Rose to know what kind of adjustment their girls make once they are back in the community. This would also have implications for their treatment program.

By closely following the adjustment of the girls, the Villa st. Rose staff would be able to more clearly appraise the strengths and weaknesses of their program. That is, follow-up would help in determining what parts were most useful for the girls once released and which were least useful. With this knowledge, Villa st. Rose would be very able to plan to meet as fully as possible the needs of each girl in treatment so as to help her more fully realize her potential.

Several studies in the chapter on Review of the Literature have pointed out the importance of the family relationships to the adolescent. One of the findings of our study was that the 
mother tended to perceive the daughter's attitude rather accurately, which could mean that, of the family members, the mother tries to keep a close relationship with the daughter. Looking at the family as a significant factor then in the girl's life, despite the fact that many do not live at home after release, could mean that follow-up should be done not only with the girl but also with the family. Besides providing help to the girl and her family, it would also provide Villa st. Rose with a maximum of information regarding their treatment program. Perhaps one of the weakest aspects of the program is the lack of financial resources available for follow-up care. It appears that it would be in the interest of Villa St. Rose to obtain money for such purposes in order to facilitate providing the best possible service to the girls.

RECOMMENDATIONS FOR FUTURE STUDY:

Several possibilities exist for future studies. One is a type not concerned with follow-up but with studying various aspects of the present population of Villa St. Rose. This could be concerned with the characteristics of population served such as age, economic status, geographic location, and family situation, with the treatment program in progress, or with a wide range of other possibilities. As noted before, the data would be easier to collect.

Another possibility would be a study following the girls 
for a six-month period immediately after release. The girls would be selected at the time of their release for the study and informed of the research plans. A questionnaire could be developed for them to complete at the time of release and again six months later. The responses would then be compared. Or the girls could be told they would be contacted six months or a year later for a single follow-up attempt. A follow-up study such as the present one could be done with individual questionnaires not set up for comparison.

There are other research possibilities but it is not necessary to delineate them here. However, one should be aware of the large numbers of problems which can be encountered in doing such studies. We hope that by our detailing of the problems encountered in this study, others will be helped to avoid having the same experience. We realize that this study is not without mistakes, but we have learned from them. 
FOOTNOTES 
FOOTNOTES

CHAPTER I

${ }^{1}$ David street, Robert Vinter, Charles Perrow, Organization for Treatment, (New York: The Free Press, 1966), pp. 3-4.

CHAPTER IV

2 Sigmund Freud, A General Instroduction to Psychoanalysis (New York: Permabooks, 1953).

${ }^{3}$ Erik Erikson, Childhood and Society (New York: W. W. Norton and Company, Inc., 1963).

${ }^{4}$ Ibid.

${ }^{5}$ Ibid.

${ }^{6}$ Ibid.

7 Margaret Mead, "Adolescence in Primitive and Modern Society," Readings In Social Psychology, eds., Swanson, Newcomb, and Hartley, (New York: Henry Holt, 1952).

${ }^{8}$ E. z. Friendenberg, The Vanishing Adolescent (Boston: Beacon Press, 1959).

${ }^{9}$ Ibid. , p. 13.

${ }^{10} \mathrm{Clifford}$ Shaw, Delinquency Areas (Chicago: University of Chicago Press, $1 9 5 9 \longdiv { \text { . } }$

llWilliam Kvaraceus and Walter B. Miller, Delinquent Behavior, Culture, and the Individual (Washington: National Educational Association of the United states, 1959). 
12 Ralph W. England, Jr., "A Theory of Middle-Class Juvenile Delinquency," Journal of Criminal Law, Criminology, and Police Science, 50 (March-April, 1960), pp. 535-540.

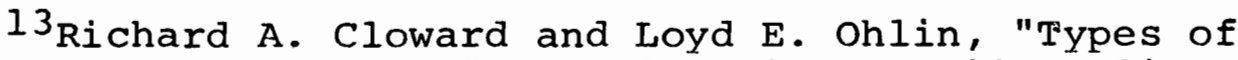
Delinquent Subcultures," Readings in Juvenile Delinquency, ed. Ruth Cavan (New York: J. B. Lippincott Company, 1964) pp. 90-111.

14Sidney Berman, "Antisocial Character Disorder: Its Etiology and Relationship to Delinquency," American Journal of Orthopsychiatry, 29 (1959), pp. 612-621.

$15_{A}$ lbert $\mathrm{K}$. Cohen, "The Delinquent Subculture," Readings In Juvenile Delinguency, ed. Ruth Cavan (New York: J. B. Lippincott Company, 1964), pp. 75-90.

16 Charles J. Browning, "Toward a Science of Delinquency Analysis," Sociology and Social Research, 46 (1961-1962), pp. 61-74.

$$
\begin{aligned}
& { }^{17} \text { Ibid. , p. } 64 . \\
& { }^{18} \text { Ibid. , p. } 65 .
\end{aligned}
$$

${ }^{19}$ Committee on Continuing Legal Education, Oregon Juvenile Law Handbook (Oregon: Oregon State Bar, 1970), Chapter 1, Section 1.1.

${ }^{20}$ Ibid., Chapter 5, Section 5.3.

${ }^{21}$ Ibid., Chapter 5, Section 5.3.

$22 \mathrm{Dr}$. John Matsushima, "Some Aspects of Defining "Success" in Residential Treatment," Child Welfare, Vol. 44, No. 5, (1965), p. 272 .

$23^{\text {Ibid. , p. } 272 .}$ 
24 Ibid.

25Lerman, Paul, "Evaluative Studies of Institutions for Delinquents: Implications for Research and Social Policy", Social Work, Vol. 13, No. 3, (1968), pp. 55-64.

${ }^{26}$ Ibid. , p. 63.

27Sidney Aronson, and Clarence Sherwood, "Researcher vs. Practitioner", Social Work, Vol. 12, No. 4, (1967), pp. 89-96.

${ }^{28}$ Ibid. , p. 95.

${ }^{29}$ Ibid., p. 96.

30 Sister Mary of St. William (McGlone), "Some Characteristics of 189 Girls who Terminated Residence In The Home of the Good Shepherd in an Eighteen Month Period Between June 30, 1947 and December 31, 1948, and Their Present Status After A Five Year Period", Unpublished Thesis, University of Washington, Seattle, Washington, (1954), p. 44 .

31"Character Education in the Home of the Good Shepherd," Unpublished Thesis, Seattle University, (1954), p. 45.

32Thomas DeJardin, et al., "Assessment of subjective Experiences of Boys Entering the Community From A Correctional School," Unpublished Thesis, School of Social Work, Portland State University, (1968), p. 73 .

${ }^{33}$ Ibid. , p. 74 .

${ }^{34}$ James Cowden, and Asher Pacht, "Predicting Institutional and Post-Release Adjustment of Delinquent Boys," Journal of Consulting Psychology, Vol. 31, No. 4, (1967), pp. 377.

${ }^{35}$ Ibid. , p. 380. 
36 James Cowden, "Predicting Institutional Adjustment and Recidivism in Delinquent Boys", Journal of Criminal Law, Criminology and Police Science, Vol. 57, (1966), p. 44 .

37Dr. Delore Taylor, and Phillip Starr, "Foster Parenting: An Integrative Review of the Literature", Child Welfare, Vol. 46, No. 7, (1967), pp. 371-385.

38Harriet Snyderwan, "Application of Research Finding in Relation to Foster Care", Child Welfare, Vol. 46, No.7, (1968), pp. 386-392.

${ }^{39}$ Eleanor Glueck, and James Cheldon, Unraveling Juvenile Delinquency, New York: The Hildreth Press, Inc., (1950), p. 130 .

40 Jon Simpson, and Maurice D. Van Arsdol, Jr., "Residential History and Educational Status of Delinquents and NonDelinquents", Social Problems, Vol. 15, No. 1, (1967), pp. 2540 .

$41_{R}$. E. Passingham, "study of Delinquents with Children's Home Background", British Journal of Criminology, Vol. 8, No. 1, (1968), pp. 32-45.

${ }^{42}$ Charles Willie, "The Relative Contribution of Family Status and Economic Status to Juvenile Delinquency", Social Problems, Vol. 14, No. 3, (1967), pp. 326-334.

43 Martin Gold, "Undetected Delinquent Behavior", Journal of Research in Crime and Delinguency, Vol. 3, No. 1, (January, 1966), pp. 27-46.

${ }^{44}$ Ibid., p. 45 .

${ }^{45}$ Simpson and Van Arsdol, Jr., op. cit., pp. 25-40.

${ }^{46}$ Donald Allen and Harjit Sandhu," A Comparative Study of Delinquents and Non-Delinquents: Family Affect, Religion, and Personal Income", Social Forces, Vol. 46, (1967-68). 
47Walter Reckless, et al.," "Study of self-Concept as an Insulator Against Delinquency", American Sociological Review, Vo1. 21, No. 6 (December 1956), pp. 744-746.

\section{CHAPTER V}

${ }^{48}$ Committee on Continuing Legal Education, 'Oregon Juvenile Law Handbook, op. cit., Chapter 1, Section 1.1.

${ }^{49}$ Mead, op. cit.

50 Erikson, op. cit.

51 Cohen, op. cit., pp. 75-90.

52Simpson and Van Arsdol, Jr., op. cit., pp. 25-40.

$$
\text { CHAPTER VI }
$$

53 Matsushima, op. cit., p. 272 .

${ }^{54}$ Aronson and Sherwood, op. cit., pp. 89-96. 
A SELECTED BIBLIOGRAPHY 


\section{A SELECTED BIBLIOGRAPHY}

Allen, Donald and Harjit Sandhu. "A Comparative study of Delinquents and Non-Delinquents," Social Problems, Vol. 15, No. 1 (1967), pp. 25-40.

Aronson, Sidney, and Clarence Sherwood. "Researcher vs. Practitioner", Social Work, Vol. 13, No. 3 (1968), pp. 55-64.

Berman, Sidney. "Antisocial Character Disorder: Its Etiology and Relationship to Delinquency." American Journal of Orthopsychiatry, Vol. 29 (1959), pp. 612-621.

Blalock, Herbert. Introduction to Social Research. New Jersey: Prentice Hall, Inc., 1970.

Browning, Charles J. "Toward a Science of Delinquency Analysis", Sociology and Social Research, Vol. 46, (1961-1962), pp. 61-74.

Cloward, Richard A., and Lloyd E. Ohlin. "Types of Delinquent Subcultures", Readings in Juvenile Delinguency, ed. Ruth Cavan. New York: J. B. Lippencott Co., (1964), pp. 75-90.

Cohen, Albert $K$. "The Delinquent Subculture", Readings in Juvenile Delinguency, ed. Ruth Cavan, New York: J. B. Lippencott Co., (1964), pp. 75-90.

Committee on Continuing Legal Education. Oregon Juvenile Law Handbook. Oregon: Oregon State Bar, (1970).

Cowden, James and Asher Pacht. "Predicting Institutional Adjustment and Recidivism in Delinquent Boys", Journal of Criminal Law, Criminology, and Police Science, Vol. 57 , (1966)

Dal1, Adolin G. "Groups Learning For Foster Parents in a Public Agency". Children, Vol. 14, No. 5 (1967), pp. 185-187. 
DeJardin, Thomas, et al. "Assessment of Subjective Experiences of Boys Entering the Community from a Correctionaj. School". Unpublished Thesis. Portland State University: School of Social Work, 1968.

Engh, J. "Help for Troubled Girls". Parents Magazine, 45 (1970), pp. 66-68.

England, Ralph W., Jr. "A Theory of Middle-Class Juvenile Delinquency". Journal of Criminal Law, Criminology, and Police Science, 50 (March-April, 1960), pp. 535-540.

Erikson, Erik. Childhood and Society. New York: W. W. Norton and Company, Inc., 1963.

Finkelstein, Harry. "Limitations in Residential Treatment". Crime and Delinquency, 14, No. 3 (1968), pp. 244-254.

Freud, Sigmund. A General Introduction to Psychoanalysis. New York: Permabooks, 1953.

McGlone, Sister Mary of St. William. "Some Characteristics of 189 Girls Who Terminated Residence in the Home of the Good Shepherd in an Eighteen Month Period Between June 30, 1947 and December 31, 1948, and Their Present Status After a Five Year Period." Unpublished Master's Thesis. Seattle: University of Washington, 1954.

Mead, Margaret. "Adolescence in Primitive and Modern Society". Readings in Social Psychology. Ed. Swanson, Newcomb, and Hartley. New York: Henry Holt, 1952.

Muuss, Ralph E. Theories of Adolescence, New York: Random House, 1968.

Passingham, R. E. "Study of Delinquents with Children's Home Background". British Journal of Criminology, 8, No. 1 (1968), pp. 32-45.

Perrow, Charles, David Street, and Robert D. Vinter. Organization for Treatment. New York: The Free Press, 1960. 
Pike, Agnew and Sandra. The Science Game. New Jersey: Prenteice Hall, 1969 .

Reckless, Walter, et al., "Study of Self-Concept as an Insulator Against Delinquency". American Sociological Review, 21, No. 6 (1956), pp. 744-746.

Schwitzelbel, Daeph. Street Corner Research. Cambridge: Harvard University, 1964.

Shaw, Clifford. Delinquency Areas. Chicago: University of Chicago Press, 1959.

Simpson, Jon and Maurice D. Van Arsdol, Jr. "Residential History and Educational Status of Delinquents and NonDelinquents." Social Problems, 15, No. 1, (1967), pp. 25-40.

Snyderwan, Harriet. "Application of Research Finding in Relation to Foster Care". Child Welfare, 46, No. 7 (1968), pp. 386-392.

Taylor, A. J. W. "An Evaluation of Group Psychotherapy in a Girl's Borstal". International Journal of Group Psychotherapy, 17, No. 2 (1967), pp. 168-177.

Volkman, Arthur P. "A Matched Group Person Comparison of Delinquent and Non-Delinquent Juveniles", Social Problems, 6, No. 3 (1958-1959), pp. 238-245.

Willie, Charles. "The Relative Contribution of Family Status and Economic Status to Juvenile Delinquency." Social Problems, 14, No. 3 (1967), pp. 326-334. 
APPENDIX I

GIRL'S CORRESPONDENCE, QUESTIONNAIRE, AND DATA 
Dear

Villa Saint Rose hopes to provide a continually improving program for its girls. Toward this end Portland State University School of Social Work Students are conducting a research project. They will be sending questionnaires to selected Villa saint Rose graduates and their parents. There will be particular emphasis" on the girls' present adjustments and how Villa saint Rose may or may not have contributed to these adjustments.

You have been chosen as one of the participants in this study. We will be sending questionnaires to both girls and parents within a few weeks, and will contact you shortly by phone to verify your present address. We will later contact you by telephane to set up a time to collect the questionnaires and answer any questions you may have concerning our research project. Your cooperation will be greatly appreciated. All information received will be confidential.

Sincerely,

Villa Saint Rose Research Group

Marie Ades

Carol Bell

Kathleen Christenson

Shirley Groves

Paula Murray 
Villa st. Rose

Research Group

597 N. Dekum St.

Portland, Oregon 97217

Dear

The enclosed questionnaire is designed for the purposes of a research study only and will be entirely anonymous. The members of the research group would appreciate it very much if you would kindly fill it out and return it to us. You may use the enclosed stamped, self-addressed envelope for this, or if you prefer, one of the researchers would be glad to pick it up. We will be calling you shortly to determine how you would rather do it and to answer any questions you may have regarding the study.

Your cooperation will be greatly appreciated.

Sincerely yours,

Villa st. Rose Research Group

Marie Ades

Carol Bell

Kathleen Christensen

Shirley Groves

Paula Murray 


\section{GIRL'S QUESTIONNAIRE}

Instructions: Please check only one answer for each question.

1. Are you presently living with your parents?

Yes

No

2. Did you live with your parents after your release from Villa st. Rose?

Yes Length of time
Reason for leaving (if applicable):

No Reason:

Where are you living now?

3. How would you describe your attitude toward your parents prior to your stay at VSR?

Mother:

warm, accepting (congenial, friendly, and respecting

\begin{tabular}{|c|c|}
\hline cooperative & $\begin{array}{l}\text { (willing to work together toward } \\
\text { common goals.) }\end{array}$ \\
\hline tolerant & $\begin{array}{l}\text { (recognizing and respecting the } \\
\text { other person's decisions, whether } \\
\text { agreeing with his decisions or not.) }\end{array}$ \\
\hline indifferent & $\begin{array}{l}\text { (expressing no marked feelings, } \\
\text { either negative or positive.) }\end{array}$ \\
\hline antagonistic & (having and showing strong dislike.) \\
\hline
\end{tabular}


Father:

warm, accepting (Same definitions apply as in the cooperative tolerant first part of question 3.) indifferent antagonistic

4. How would you describe your present attitude toward your parents?

Mother: warm, accepting cooperative tolerant indifferent antagonistic

(Same definitions apply as in first part of question 3 .

cooperative
tolerant
indifferent
antagonistic

Father:

warm, accepting cooperative tolerant indifferent antagonistic

5. How would you describe your attitude toward your brothers and sisters prior to VSR?

Attitude

Warm, accepting

Cooperative

Tolerant

Indifferent

Antagonistic
Brothers and Sisters

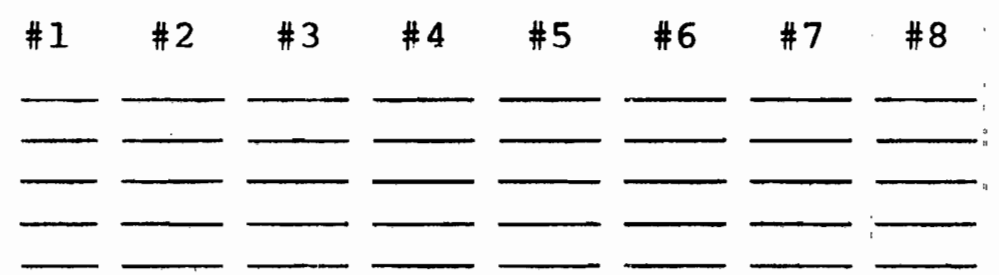

Note: If there are more brothers and sisters, please use this extra space to write them in. 
6. How would you describe your present attitude toward your brothers and sisters?

Attitude Brothers and Sisters

Warm, accepting

\#1 $\quad$ \#2 \#3 \#4 \#5 \#6 \#7 \#8

Cooperative

Tolerant

Indifferent

Antagonistic

Note: If there are more brothers and sisters, please use this extra space to write them in.

7. In your estimation how much did you discuss problems with your parents prior to VSR?

Not at all
Very little
Some
A great deal
Do not know

8. In your estimation how much do you discuss problems with them now?

Not at all

Very little
Some
A great deal
Do not know

9. To what extent do you feel that your friends had an influence on you prior to VSR?

Positive

None

Very little

some

A great deal

Do not know
Negative

None
Very little
Some
A great deal
Do not know


10. To what extent do you feel that your friends have an influence on you now?

Positive

\begin{tabular}{l} 
None \\
Very little \\
Some \\
A great deal \\
\hline Do not know
\end{tabular}

Negative

None
Very little
Some
A great deal
Do not know

11. Do you have the same friends now as you did prior to VSR? Yes
No

12. What type of experience do you feel that Villa St. Rose was for you?

very positive

Positive

Neutral

Negative

Very negative
(Significantly increased the ability to understand and to cope with oneself and with the community.)

(Increased the ability to understand and to cope with oneself and with the community.)

(No change.)

(Decreased the ability to understand and to cope with oneself and with the community.)

(Significantly decreased the ability to understand and to cope with oneself and with the community.)

13. If you could change something at VSR, what would you change?

About Yourself:

About the Program:

14. For those married: How would you describe your present relationship with your husband? 
PRE-TEST

GIRL'S QUESTIONNAIRE

\begin{tabular}{|c|c|c|c|c|c|c|c|c|}
\hline $\begin{array}{l}\text { Pre-test } \\
\text { Sample }\end{array}$ & 1 & $\begin{array}{l}3 \& 4 \\
\text { Mother }\end{array}$ & $\begin{array}{l}3 \& 4 \\
\text { Father }\end{array}$ & $5 \& 6$ & $7 \& 8$ & $\begin{array}{l}\text { Compo- } \\
\text { site } \\
\text { score }\end{array}$ & $9 \& 10$ & 12 \\
\hline 1 & $\begin{array}{l}\mathrm{N} \\
\mathrm{Y}\end{array}$ & - & - & - & - & - & - & - \\
\hline 2 & $\begin{array}{l}Y \\
Y\end{array}$ & 1 & NS & 0 & 1 & 2 & 3 & 4 \\
\hline 3 & $\begin{array}{l}\mathbf{N} \\
\mathrm{N}\end{array}$ & 0 & 0 & 0 & 1 & $I$ & -2 & 2 \\
\hline 4 & $\begin{array}{l}Y \\
Y\end{array}$ & 4 & 0 & 0 & 2 & 6 & -2 & 1 \\
\hline 5 & $\begin{array}{l}\mathrm{Y} \\
\mathrm{Y}\end{array}$ & 2 & 2 & 0 & 0 & 4 & 3 & 5 \\
\hline 6 & $\begin{array}{l}\mathrm{N} \\
\mathrm{N}\end{array}$ & 3 & 3 & 3 & 3 & 12 & 1 & 5 \\
\hline
\end{tabular}

1. Question 1 was used to determine if the girl had lived at home since her release from VSR (top letter), and if she was living at home at the time of our study (bottom letter) $\mathrm{N}$ means no, $\mathrm{Y}$ means yes.

2. Questions $3 \& 4$ (mother), $3 \& 4$ (father), $5 \& 6$, and $7 \& 8$ were included in the total composite score.

3. Questions $9 \& 10$ dealt with peer influence and were used only for correlation purposes.

4. NS means no score or no response on the question. 


\section{GIRL'S QUESTIONNAIRE}

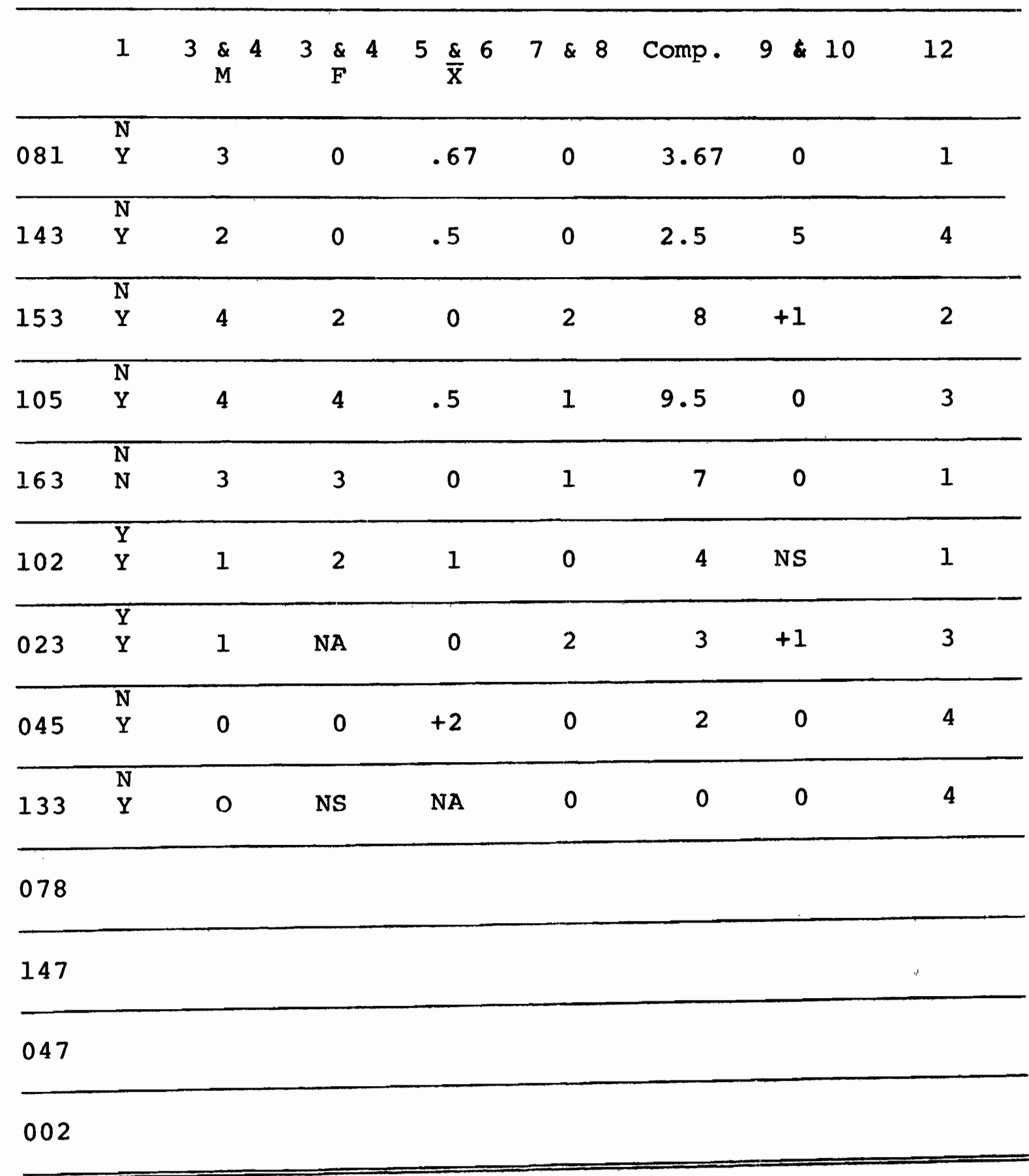




\section{APPENDIX II}

PARENT'S CORRESPONDENCE, QUESTIONNAIRE, AND DATA 
Dear

Villa saint Rose hopes to provide a continually improving program for its girls. Toward this end Portland State University School of Social Work Students are conducting a research project. They will be sending questionnaires to selected Villa Saint Rose qraduates and their parents. There will be particular emphasis on the girls' present adjustments and how Villa saint Rose may or may not have contributed to these adjustments.

Your daughter has been chosen as one of the participants in this study. We will be sending questionnaires to both girls and parents within a few weeks, and will contact you shortly by phone to verify your present address. We will later contact you by telephone to set up a time to collect the questionnaires and answer any questions you may have concerning our research project.

If your daughter is no longer living at this address, would you please forward this information to her. Your cooperation will be greatly appreciated. All information received will be confidential.

sincerely,

Villa Saint Rose Research Group

Marie Ades

Carol Bell

Kathleen Christenson

Shirley Groves

Paula Murray 
Villa st. Rose

Research Group

597 N. Dekum St.

Portland, Oregon

97217

Dear

The enclosed questionnaire is designed for the purposes of a research study only and will be entirely " anonymous. The members of the research group would appreciate it very much if you would kindly fill it out and return it to us. You may use the enclosed stamped; self-addressed envelope for this, or if you prefer, one of the researchers would be glad to pick it up. We will be calling you shortly to determine how you would rather do it and to answer any questions you may have regarding the study.

Your cooperation will be greatly appreciated.

Sincerely yours,

Villa st. Rose Research Group

Marie Ades

Carol Bell

Kathleen Christensen

Shirley froves

Paula Murray 
December 9, 1971

Dear

I have recently attempted to contact you by telephone and was unable to do so because of a number change. I am assuming that the questionnaire and previous letters have been forwarded to you at your new address.

The return of all the questionnaires is essential for the successful completion of our study, thus I would like to ask another favor of you. If it is at all possible, could you please call Villa saint Rose (285-3030), and inform them of your receipt of the letters and of the date which you will be able to have the questionnaire returned. If you would like us to pick up the questionnaire, or if you have any questions, please leave your telephone number and we will be more than happy to contact you.

Again, in the hope of improving the future program at Villa, the return of your questionnaire is essential. Your cooperation is greatly appreciated. Thank you in advance. Sincerely,

Villa Saint Rose Research Group

Marie Ades

Carol Bell

Kathleen Christensen

Shirley Groves

Paula Murray 
PARENTS' QUESTIONNAIRE

Instructions: Please check only one answer for each question.

1. Is your daughter presently living at home? Yes

No

2. Did she live at home after her release from Villa st. Rose?

$\begin{array}{ll}\text { Yes } & \text { Length of time } \\ & \text { Reason for leaving (if applicable): } \\ \text { No } & \text { Reason: }\end{array}$

Where is she now?

3. How would you describe your daughter's attitude toward you as parents prior to her stay at VSR?

Mother

warm, accepting (congenial, friendly, and respecting

$\begin{array}{ccc}\text { warm, accepting } & \begin{array}{c}\text { (congenial, friendly, and respecting } \\ \text { of the parent's own individuality.) } \\ \text { cooperative } \\ \text { (willing to work together toward } \\ \text { commongoals.) }\end{array} \\ \text { (recognizing and respecting the } \\ \text { other person's decisions, whether } \\ \text { agreeing with his decisions or not.) } \\ \text { indifferent }\end{array} \quad \begin{gathered}\text { (expressing no marked feelings, either } \\ \text { negative or positive.) } \\ \text { (having and showing strong dislike.) }\end{gathered}$


Father:

warm, accepting

(same definitions apply as in

cooperative

tolerant

the first part of question 3.)

indifferent

antagonistic

4. How would you describe her present attitude toward you as parents?

Mother:

warm, accepting

cooperative

tolerant

indifferent

(same definitions apply as in the antagonistic

first part of question 3.)

Father:

warm, accepting

cooperative

tolerant

indifferent antagonistic

5. How would you describe your daughter's attitude toward her brothers and sisters prior to her stay at VSR?

Attitude

Warm, accepting

Cooperative

Tolerant

Indifferent

Antagonistic

\section{Brothers and Sisters}

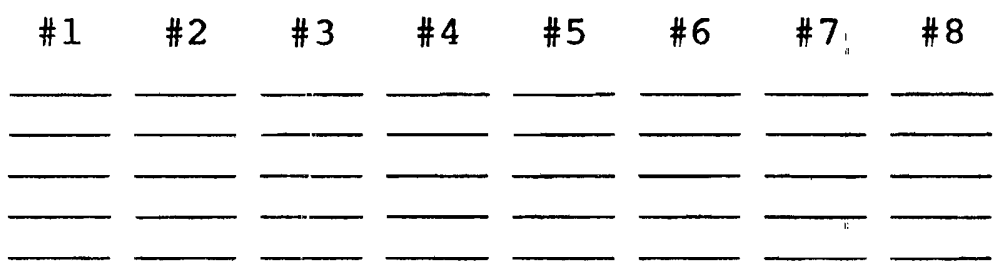

Note: If there are more brothers and sisters, please use this extra space to write them in. 
6. How would you describe her present attitude toward her brothers and sisters?

Attitude

Warm, accepting

Cooperative

Tolerant

Indifferent

Antagonistic
Brothers and Sisters

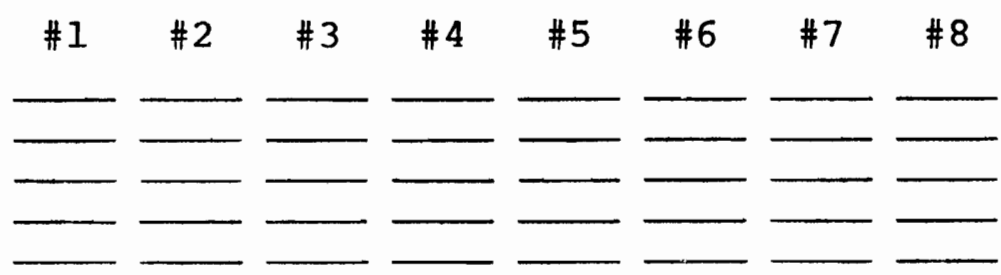

Note: If there are more brothers and sisters, please use this extra space to write them in.

7. In your estimation how much did your daughter discuss problems with you prior to VSR?

Not at all

Very little

Some

A great deal

Do not know

8. In your estimation how much does she discuss problems with you now?

Not at all

Very little

Some

A great deal

Do not know

9. To what extent do you feel that your daughter's friends had an influence on her prior to VSR?

Positive

None

Very little

Some

A great deal

Do not know
Negative

None

Very little

Some

A great deal

Do not know 
10. To what extent do you feel that your daughter's friends have an influence on her now?

Positive:

None
Very little
Some
A great deal
Do not know

Negative

None
Very little
Some
A great deal
Do not know

11. Does she have the same friends now as she did prior to VSR?

\begin{tabular}{l} 
Yes How many? \\
\hline No \\
\hline Do not know
\end{tabular}

12. What type of experience do you feel that Villa st. Rose was for your daughter?

Very positive (Significantly increased the ability to understand and to cope with oneself and with the community.)

Positive

(Increased the ability to understand and to cope with oneself and with the community.)

Neutral

Negative

Very negative
(No change.)

(Decreased the ability to understand and to cope with oneself and with the community.)

(Significantly decreased the ability to understand and to cope with oneself and with the community.) 
PRE-TEST

PARENT QUESTIONNAIRE

Pre-Test

Sample

$1 \quad 3 \& 4 \begin{array}{llllllllll}3 & \& & 4 & 5 & \& & 6 & 7 & \& & 8\end{array}$

Compo- $9 \& 10 \quad 12$

site

Score

\begin{tabular}{ccccccccc}
\hline 1 & $\mathrm{~N}$ & 0 & 1 & -1 & 0 & 0 & 0 & 3 \\
\hline 2 & $\mathrm{Y}$ & - & - & - & - & - & - & - \\
\hline 3 & $\mathrm{Y}$ & 0 & 0 & 0 & 0 & 0 & 1 & 1 \\
\hline 4 & $\mathrm{~N}$ & 4 & $\mathrm{NS}$ & 1 & 2 & 7 & -3 & 5 \\
\hline 5 & $\mathrm{Y}$ & 2 & 2 & 1 & 0 & 5 & 2 & 5 \\
\hline 6 & $\mathrm{~N}$ & 4 & 3 & 4 & 1 & 12 & 2 & 5 \\
\hline \hline
\end{tabular}

1. Question 1 was used to determine if the girl had lived at home since her release from VSR (top letter), and if she was living at home at the time of our study (bottom letter) $\mathrm{N}$ means no, $\mathrm{Y}$ means yes.

2. Questions $3 \& 4$ (mother), $3 \& 4$ (father), $5 \& 6$, and $7 \& 8$ were included in the total composite score.

3. Questions $9 \& 10$ dealt with peer influence and were used only for correlation purposes.

4. NS means no score or no response to the question. 
PARENT'S QUESTIONNAIRE

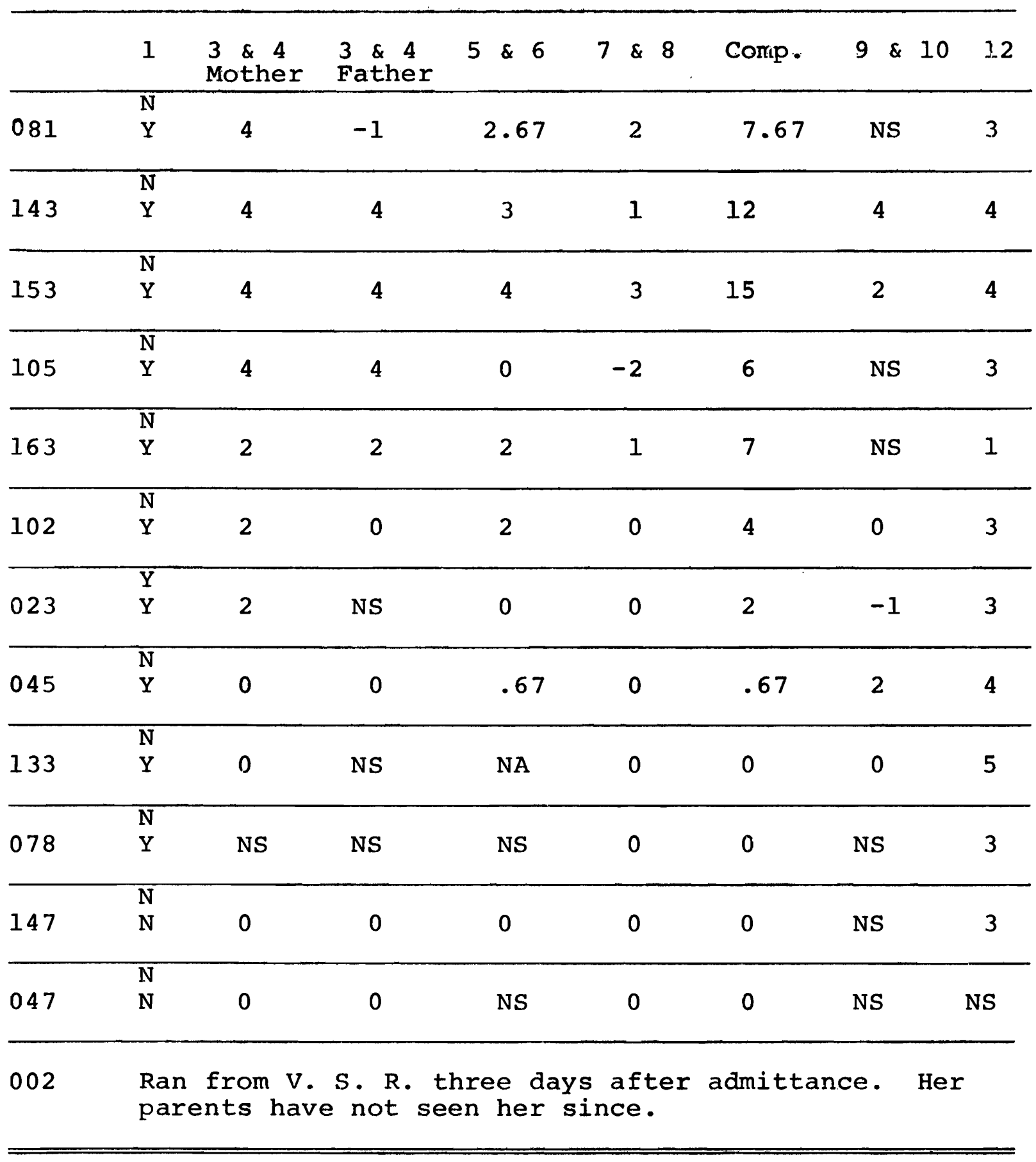


APPENDIX III

COURT'S QUESTIONNAIRE, AND DATA 
COURT QUESTIONNAIRE

The following questions should be answered according to infor:mation at the last contact with the girl and/or her family.

1. What disposition was made by the court following the girl's release from Villa st. Rose?

wardship dismissed wardship continued, no supervision wardship continued, supervision continued informal supervision

2. What has been the nature of the court's legal involvement with the girl since her release from VSR?

no further contact contact, no action taken contact, informal supervision contact, formal supervision contact, reinstitutionalization or remanded to adult court outside court's jurisdiction

3. What has been the pattern of the court's contact with the parents of this girl since her release from VSR? none weekly contacts twice a month monthly other, specify:

4. What was the parents' attitude toward the court?

Prior to commitment to VSR?

seek court assistance (actively requests court's help) cooperative (willing to work together toward common goals at the instigation of the court) tolerant (recognize and respect court's decision, whether agreeing with court or not) 
resistant (strive and work against court's decision)

antagonistic (having and showing strong do not know dislike towards the court)

Since her release from VSR?

wants court assistance (actively requests court's help)

cooperative (willing to work together toward common goals at the instigation of the court)

tolerant (recognize and respect court's decision, whether agreeing with court or not)

resistant (strive and work against court's decision)

antagonistic (having and showing strong dislike towards the court) do not know

5. What was the parents' attitude toward the girl?

Prior to commitment to VSR?

$\begin{array}{cc}\text { warm, accepting } & \begin{array}{c}\text { (congenial, friendly, and } \\ \text { respecting of the girl's } \\ \text { individuality) } \\ \text { (willing to work together } \\ \text { toward common goals) } \\ \text { (recognizing and respecting } \\ \text { the girl's decisions, } \\ \text { whether agreeing with them } \\ \text { or not) } \\ \text { (expressing no marked } \\ \text { feelings, either positive } \\ \text { or negative) } \\ \text { (having and showing strong } \\ \text { dislike toward the girl) }\end{array} \\ \text { do not know } & \end{array}$

Since her release from VSR?

warm, accepting

(same definitions as immediately above in cooperative question 5) 
tolerant

indifferent

antagonistic

do not know

6. What was the girl's attitude toward her parents?

Prior to commitment to VSR?

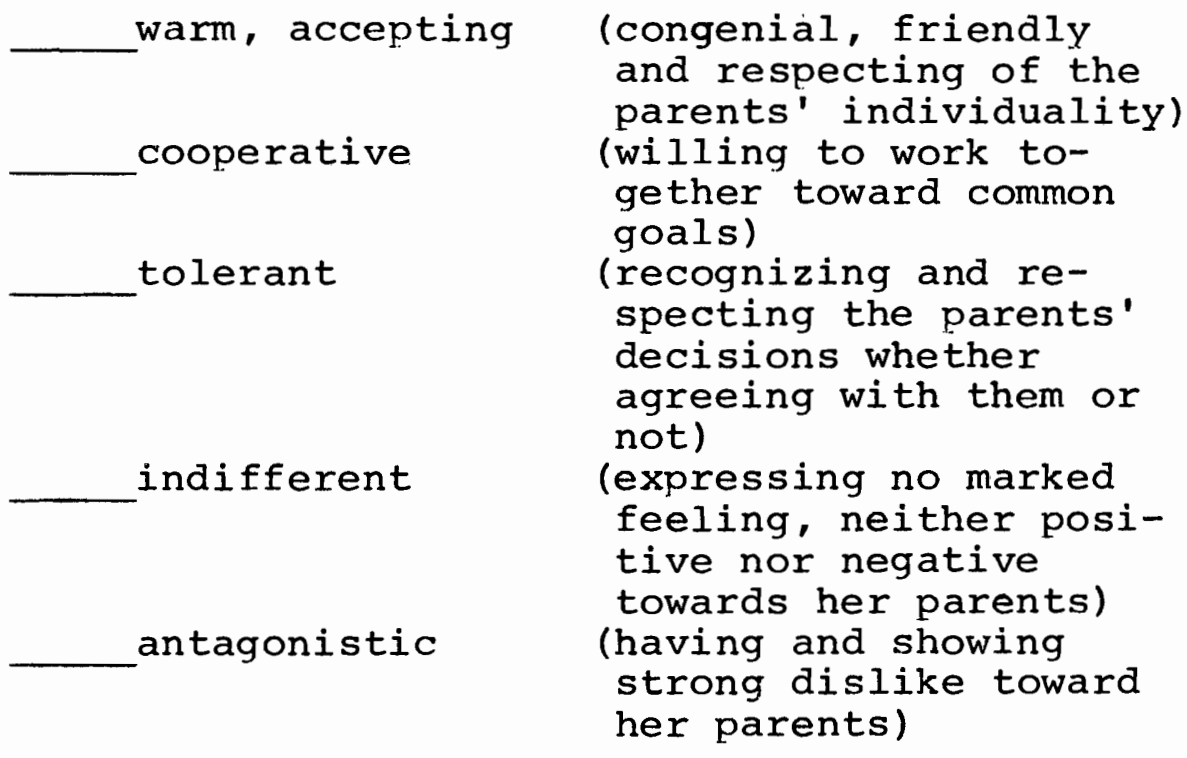

Since her release from VSR?

warm, accepting $\begin{array}{r}\text { (same definitions as } \\ \text { immediately above } \\ \text { in question 6) }\end{array}$
tolerant
indifferent
antagonistic
do not know

7. What was the peer group's influence upon this girl's illegal activities?

Prior to her commitment to VSR? 
7. (Cont'd)

attempted to prevent illegal activity

disapproved of illegal activity no influence approved of illegal activity participated in and encouraged illegal activity no peer group involvement

Since her release from VSR?

attempted to prevent illegal activity disapproved of illegal activity no influence approved of illegal activity participated in and encouraged illegal activity no peer group involvement

8. Upon the girl's release from VSR, did she continue in her relationships with the same peer group she had prior to admission to VSR?

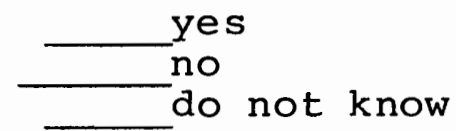

9. In your opinion is this girl presently having difficulties as a result of her peer group involvement?

yes
no
do not know

10. In your opinion this girl's social-emotional functioning has :

improved significantly
improved
no change
deteriorated
deteriorated significantly

11. Do you feel that VSR was a for this girl? very positive experience positive neutral negative very negative 
Please feel free to discuss any suggestions and criticisms you might have concerning this questionnaire. Your thoughts are most valuable and will aide us in our research project. Thank you. 
PRE-TEST

COURT QUESTIONNAIRE

\begin{tabular}{cccccccc}
\hline $\begin{array}{l}\text { Pre-test } \\
\text { Sample }\end{array}$ & 1 & 2 & 5 & 6 & $\begin{array}{l}\text { Compo- } \\
\text { site } \\
\text { Score }\end{array}$ & 11 & $\begin{array}{c}\text { Total } \\
\text { Points }\end{array}$ \\
\hline 1 & a & 5 & 4 & 3 & 12 & 5 & 13 \\
\hline 2 & $\mathrm{a}$ & 5 & 0 & 0 & 5 & 4 & 5 \\
\hline 3 & $\mathrm{c}$ & -5 & 0 & 2 & -3 & 3 & 13 \\
\hline 4 & $\mathrm{a}$ & 5 & 0 & 1 & 6 & 3 & 13 \\
\hline 5 & $\mathrm{~b}$ & 3 & 0 & 1 & 4 & 3 & 13 \\
\hline 6 & $\mathrm{c}$ & 5 & 1 & 0 & 6 & 2 & 13 \\
\hline
\end{tabular}

1. Questions 2, 5 and 6 were the only questions included in the composite score.

2. Question 1 referred to the behavior of the court after resuming jurisdiction upon the girl's release from villa St. Rose. Therefore no numerical value was placed on it. Letters in descending order were used to identify the action taken by the court.

3. The total points possible indicates the number of points a girl could have received in her composite score. The total for each girl was limited by the number of questions the court was able to answer.

4. NS means no score or no response by the court. 


\section{COURT QUESTIONNAIRE}

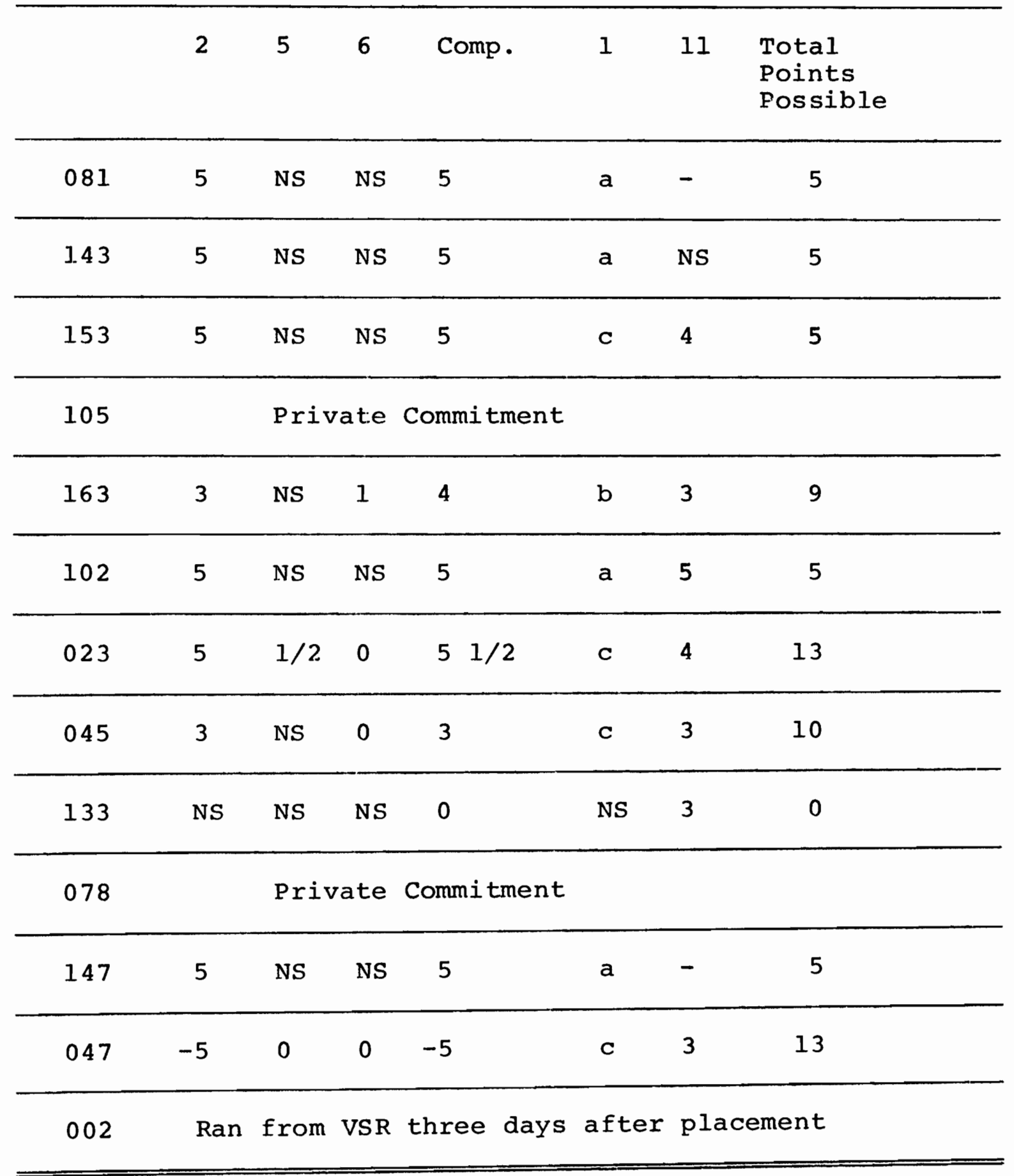


COURT QUESTIONNAIRES

\begin{tabular}{|c|c|c|c|c|c|c|c|}
\hline & 2 & 5 & 6 & Comp. & 1 & 11 & $\begin{array}{l}\text { Total pts } \\
\text { Possible }\end{array}$ \\
\hline 040 & 5 & NS & NS & 5 & $\mathbf{b}$ & - & 5 \\
\hline 136 & 3 & NS & NS & 3 & $\mathrm{~b}$ & 3 & 5 \\
\hline 139 & 5 & NS & NS & 5 & $\mathrm{a}$ & 4 & 5 \\
\hline 099 & 5 & NS & NS & 5 & $a$ & 4 & 5 \\
\hline 073 & - & - & - & - & - & - & - \\
\hline 079 & -3 & 1 & 2 & 0 & C & 4 & 13 \\
\hline 091 & 3 & 1 & 3 & 7 & $\mathrm{~b}$ & 5 & 13 \\
\hline 151 & 5 & 0 & -1 & 4 & $a$ & 3 & 13 \\
\hline 058 & 5 & 0 & NS & 5 & $\mathrm{~b}$ & NS & 9 \\
\hline 129 & 5 & NS & NS & 5 & $a$ & NS & 5 \\
\hline 170 & 5 & NS & NS & 5 & $a$ & NS & 5 \\
\hline 141 & 5 & NS & NS & 5 & $\mathrm{~b}$ & NS & 5 \\
\hline 113 & 5 & NS & NS & 5 & $a$ & $\mathrm{NS}$ & 5 \\
\hline 061 & 5 & NS & NS & 5 & $a$ & NS & 5 \\
\hline 034 & 5 & NS & NS & 5 & C & 5 & 5 \\
\hline 016 & -5 & -1 & 2 & -3 & c & 3 & 13 \\
\hline
\end{tabular}


APPENDIX I.V

SCHOOL QUESTIONNAIRE 


\section{SCHOOL QUESTIONNAIRE}

1. What was this student!s grade point average for the year prior to her commitment to Villa st. Rose?

2. What was her grade point average for the year following her release from Villa st. Rose?

3. How would you rate her attitude toward her teachers and other authority figures for the year prior to her commitment to Villa st. Rose?

warm, accepting (congenial, friendly, and respecting of the other's individuality)

cooperative

tolerant

(willing to work together toward common goals)

(recognizing and respecting the other person's decisions, whether agreeing with them or not)

indifferent

(expressing no marked feelings, either negative or positive)

antagonistic

(having and showing strong dislike) do not know

4. How would you rate her attitude toward her teachers and other authority figures in the year following her release?

$\begin{array}{ll}\text { warm, accepting } & \begin{array}{c}\text { (same definitions apply as in } \\ \text { question three) } \\ \text { cooperative } \\ \text { tolerant }\end{array} \\ \text { indifferent } & \\ \text { antagonistic } & \\ \text { do not know } & \end{array}$

5. What was her attitude toward her peers in the year prior to her commitment to Villa st. Rose?

warm, accepting (same definitions apply as in cooperative question three)

tolerant

indifferent hostile, rejecting do not know 
6. What was her attitude toward her peers in the year following her release from Villa St. Rose?

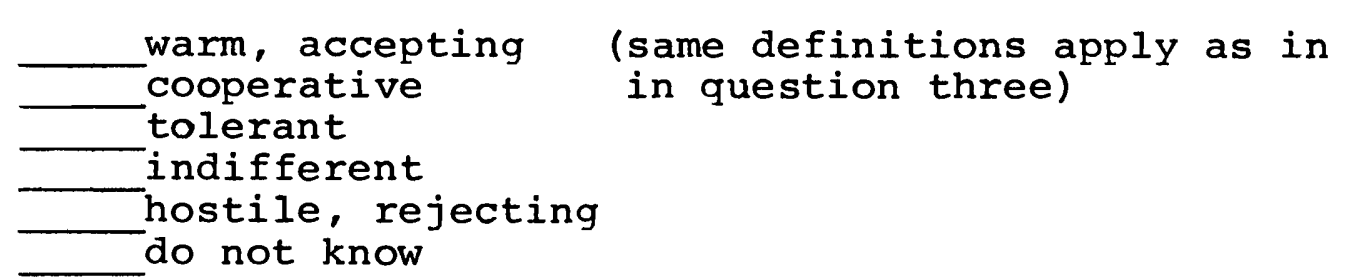

7. What was the peer group's influence upon this girl's school behavior for the year prior to her commitment to Villa St. Rose?

attempted to prevent violation of school rules disapproved of violation of school rules no influence encouraged violation of school rules participated in or approved of violation of school rules do not know

8. What was the peer group's influence upon this girl's school behavior in the year following her release from Villa St. Rose?

attempted to prevent violation of school rules disapproved of violation of school rules no influence encouraged violation of school rules participated in or approved of violation of school rules do not know

9. Did she participate in any school club or activities for the year prior to her commitment to Villa st. Rose?

yes
no
do not know
If yes, please specify:
school clubs
dances
sports
student government
sother


10. Did she participate in any school clubs or activities in the year following her release from Villa st. Rose?

yes
no
do not know
If yes, please specify:
school clubs
dances
sports
student government
other

